\title{
TWO-DIMENSIONAL MODELING OF MATERIAL FAILURE IN REINFORCED CONCRETE BY MEANS OF A CONTINUUM STRONG DISCONTINUITY APPROACH
}

\author{
J. Oliver 1, D.L. Linero ${ }^{2}$, A.E. Huespe ${ }^{3}$, O. L. Manzoli 4 \\ ${ }^{1}$ E.T.S. d'Enginyers de Camins, Canals i Ports, Technical University of Catalonia (UPC) \\ Campus Nord UPC, Mòdul C-1, c/Jordi Girona 1-3, 08034 Barcelona, Spain \\ ${ }^{2}$ Engineering School, National University of Colombia, Bogotá, Colombia \\ ${ }^{3}$ CIMEC/Intec, Conicet, Guemes 3450, Santa Fe 3000, Argentina \\ ${ }^{4}$ Department of Civil Engineering, Sao Paulo State University (UNESP), \\ Av. Luiz Edmundo C. Coube, S/N, 17030-360, Bauru, SP, Brazil \\ e-mail: xavier.oliver@upc.edu
}

Key words: computational material failure, reinforced concrete, mixture theory, continuum strong discontinuity approach

\begin{abstract}
The paper presents a new methodology to model material failure, in twodimensional reinforced concrete members, using the Continuum Strong Discontinuity Approach (CSDA). The Hill's mixture theory is used as the methodological approach to model reinforced concrete as a composite material, constituted by a plain concrete matrix reinforced with two embedded orthogonal long fiber bundles (rebars). Matrix failure is modeled on the basis of a continuum damage model, equipped with strain softening, whereas the rebars effects are modeled by means of phenomenological constitutive models devised to reproduce the axial non-linear behavior, as well as the bond-slip and dowel effects. The proposed methodology extends the fundamental ingredients of the standard Strong Discontinuity Approach, and the embedded discontinuity finite element formulations, in homogeneous materials, to matrix/fiber composite materials, as reinforced concrete. The specific aspects of the material failure modeling for those composites are also addressed. A number of available experimental tests are reproduced in order to illustrate the feasibility of the proposed methodology.
\end{abstract}

\section{MOTIVATION}

As it is well known, the mechanical behavior of reinforced concrete structural members is very affected by the formation of cracks during loading. In many situations, the ultimate loading capacity of these members is determined by a collapse mechanism induced by the formation of one or several dominant cracks, which, however, develop as concrete is already very damaged by previous secondary cracking. Therefore, in order to model the ultimate (post-critical) behavior of reinforced concrete members it is fundamental to have available numerical tools capable to appropriately describe the formation and propagation of multiple cracks in a non-homogeneous (composite) solid, constituted by a plain concrete matrix with steel reinforcement embedded in it.

Despite the important progress achieved in the last years, modeling formation and propagation of discontinuities in solids still remains an open issue in computational mechanics. The major obstacles to be overcome are related to the multiple scale character of those problems, involving the formation of tiny dissipative zones preceding the cracks (crack process zones), and the material instabilities caused by the progressive deterioration of the mechanical properties in these zones. 
In the context of the Finite Element Method (FEM), the use of local constitutive models to describe the deterioration of the mechanical properties, characterizing the crack formation, yields, in many cases, spurious mesh-size and mesh-bias dependences. The popular smeared crack models [24, 37], based on orthotropic constitutive models derived from the assumption that the cracks are smeared over an element, are also amenable to the mentioned mesh dependences. Dependencies on the finite element size can be overcome if the softening law is related to the element size, as in the crack band method [2, 19]. However, this procedure does not preclude the mesh alignment sensitivity of the results. Despite this, and possibly due to the lack of other alternatives, in current practice cracking in reinforced concrete is nowadays predominantly modeled with smeared crack models.

On the other hand, in discrete interface models cracks are modeled by interface (zerothickness) elements placed between two adjacent solid finite elements. The material degradation in the crack process zone is captured at those elements by means of discrete constitutive models, relating cohesive stresses and relative displacements (crack opening and sliding), belonging to the fictitious crack models introduced by Hillerborg [10]. Using discrete interface models to capture arbitrary crack propagation is not an easy task. The mesh topology must be continuously updated during the analysis in order to adjust the sides of the solid elements to the propagating crack surface. This type of approach does not exhibit the mesh dependence of the smeared crack model, but demands very sophisticated automated mesh regeneration techniques $[1,6]$.

An alternative way for capturing arbitrary cracks is based on a fixed mesh in which all the solid elements are surrounded by cohesive interface surfaces [42, 45]. Some encouraging results have been obtained with this methodology by Pandolfi and Ortiz [34]. However, the results can exhibit mesh alignment sensitivity.

In addition, the objectivity problems found in the methodologies described so far can be incremented in reinforced concrete problems, exhibiting multiple crack patterns. Those difficulties can be alleviated by resorting to the concept of strong discontinuity and to the so-called embedded strong discontinuity finite elements (E-FEM) [8, 16, 21], or to the extended finite elements (X-FEM) [3, 44] which allow introducing a displacement discontinuity into the finite element, independently of its boundaries. This ability makes this class of approaches very appealing to capture arbitrary crack propagation with fixed finite element meshes without loss of mesh objectivity.

A specific formulation of this family, the Continuum Strong Discontinuity Approach (CSDA) [17, 20, 23, 25, 39], is of interest here. In this approach, instead of using an explicit fictitious crack model for modeling the constitutive behavior at the embedded discontinuity interface (strong discontinuity), a continuum type (stress vs. strain) constitutive model is used. Then, the so called strong discontinuity kinematics projects that model onto the interface as a traction-separation law. This procedure seems especially suitable when it is intended to model material failure in composite materials, as modeling some phenomena is easier via continuum constitutive models.

As a crucial ingredient, the CSDA requires the use of tracking algorithms that ensure continuity of the crack path when passing from one element to another $[12,30]$. In initial stages of its development, local tracking algorithms limited the application to 
cases involving propagation of just one or very few cracks. More recently, global tracking algorithms for multiple cracks simultaneously developing, have been proposed $[25,31]$, extending the range of applicability of the embedded crack elements to larger and more realistic structures in $2 \mathrm{D}$ and $3 \mathrm{D}$ settings.

Another issue, always present in simulations involving multiples cracks, concerns the computational robustness. As it is well known, in material failure simulations numerical instabilities, inherent to the presence of the strain or displacement softening in the constitutive models, appear mainly in the post-critical branch of the structural behavior. Moreover, formation of new cracks can deactivate the ones existing in their neighborhood. This interaction between cracks can be another important source of instabilities, which can translate into loss of robustness and lack of convergence of the non-linear computations. Related to this issue, recent advances to improve stability and robustness of material failure simulations, based on the CSDA, have been presented in references [5, 27], in which, to face convergence problems, an implicit-explicit integration scheme of the constitutive model, that leads to a positive definite constitutive operator, is proposed. A specific arc-length method is also proposed to improve the controllability of the time advancing algorithm.

With the aforementioned recent developments, the material failure modeling using the CSDA exhibits the necessary maturity to be applicable to reinforced concrete, provided that the effects of rebars in plain concrete are properly included. These effects should reflect the mechanical behavior of the steel bars, usually described by a different constitutive law, as well as the interfacial interaction between concrete and bars (bondslip and dowel action).

A mesoscopic scale treatment could be done by using homogenous elements of concrete and reinforcement, with appropriate interface elements between them to account for their mutual interaction. However, for practical reinforced concrete problems, this mesoscopic approach would demand a very large computational capacity. On the contrary, in a macroscopic scale treatment, rebars could be assumed embedded into solid finite elements, allowing the use of coarser meshes and reducing the computational costs [11].

The mixture theory [43] also offers a suitable framework to account for the reinforcement in a macroscopic level. Using this theory, fibers (rebars) can be mixed up with the matrix material (plain concrete). If fibers are long enough, as it is the case for rebars, a parallel mechanical mixture scheme, assuming that all the constituents share the same strain field (or specific components of it), can be adopted. Then, the composite material stress field can be evaluated as the (weighted) sum of the stresses returned from every individual constituent constitutive model. A similar approach can be found in reference [9]. To formulate those constitutive models, one can resort to the available, and well established, phenomenological models based on standard continuum theories and, then, the CSDA appears as a natural setting to account for the material failure of the composite material.

In this scenario, this paper proposes a new methodology to model material failure in two-dimensional reinforced concrete members by means of the CSDA. Mixture theory concepts are used to model reinforced concrete as a composite material constituted of a plain concrete matrix with long fibers bundles, oriented in two orthogonal directions, 
embedded in it. The effects of the plain concrete matrix and the individual rebars in the composite material are captured by phenomenological continuum constitutive models devised to reproduce the 2D material failure of the matrix, the axial non-linear behavior of the fibers, as well as the fiber/matrix bond-slip and dowel effects. Since the treatment of the composite material is totally done at the constitutive level, the proposed methodology preserves the fundamental ingredients of the standard formulation of the CSDA for homogeneous materials [5, 25, 27]. Because of this, this paper focuses on the constitutive models assumed for the constituents and the compatibility conditions chosen to define the composite. In order to obtain information about the propagation of the cracks, some specific aspects related to the material bifurcation analysis of the resulting composite material are also addressed. Finally, numerical simulations of existing experimental tests are presented to illustrate the capabilities of the proposed methodology.

\section{COMPOSITE MATERIAL MODEL}

In the proposed approach, reinforced concrete is assumed to be a composite material constituted by a matrix and two orthogonal long fiber bundles oriented in $\mathbf{r}$ and $\mathbf{s}$ directions (see Figure 1). According to the basic hypothesis of the mixture theory, a composite is a continuum in which each infinitesimal volume is occupied simultaneously by all constituents [43]. Assuming a parallel mechanical system, all constituents are subjected to the composite strains and the corresponding composite stresses are given by the weighted (in terms of the volume fraction) sum of the stresses of each constituent. As a consequence, the matrix strain field, $\varepsilon^{m}$, and the composite strain field, $\varepsilon$, are identical. The axial fiber strain corresponds to the component $\varepsilon^{f r}=\mathbf{r} \cdot \boldsymbol{\varepsilon} \cdot \mathbf{r}$ for fiber $r$ (in direction $\mathbf{r}$ ) and $\varepsilon^{f_{s}}=\mathbf{s} \cdot \boldsymbol{\varepsilon} \cdot \mathbf{s}$ for fiber $s$ (in direction $\mathbf{s}$ ), while the fiber angular strain is given by $\gamma^{f}=2 \mathbf{r} \cdot \boldsymbol{\varepsilon} \cdot \mathbf{s}$. Supra-indices $r, f r$ and $f s$ refer to the matrix and the fibers oriented in $\mathbf{r}$ and $\mathbf{s}$ directions, respectively.

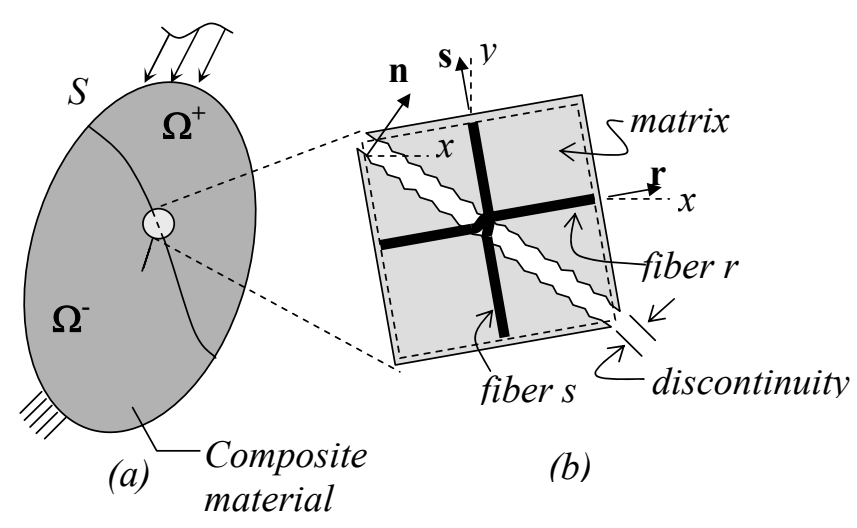

Figure 1. (a) solid with a discontinuity surface $S$, (b) representative material point of the composite material.

Then, according to the assumed model, the composite material stress tensor is computed as the sum of the stresses of each constituent, $(\bullet)$, weighted by the corresponding volumetric fraction $k^{(\bullet)}$ : 


$$
\begin{aligned}
\sigma(\varepsilon)= & k^{m} \sigma^{m}(\boldsymbol{\varepsilon})+k^{f r} \sigma^{f}\left(\varepsilon^{f r}\right)(\mathbf{r} \otimes \mathbf{r})+ \\
& +k^{f s} \sigma^{f}\left(\varepsilon^{f s}\right)(\mathbf{s} \otimes \mathbf{s})+2 \tau^{f}\left(\gamma^{f}\right)\left(k^{f r}+k^{f s}\right)(\mathbf{r} \otimes \mathbf{s})^{S}
\end{aligned}
$$

where $\sigma^{m}(\bullet), \sigma^{f}(\bullet)$ and $\tau^{f}(\bullet)$ stand for the matrix and fibers constitutive relations, returning the stresses from the corresponding strains. As we will see later, the last component of the stress field in equation (1) corresponds to the contribution due to the dowel action.

The rate form of the resulting composite constitutive equation can be written as:

$$
\dot{\sigma}=\mathbf{C}_{t g}: \dot{\varepsilon}
$$

where the constitutive tangent tensor, $\mathbf{C}_{t g}$, can be obtained by substitution of the constitutive relations of the constituents into the rate form of Equation (1), yielding:

$$
\begin{aligned}
\mathbf{C}_{t g}= & k^{m} \mathbf{C}_{t g}^{m}+k^{f r} E_{t g}^{f r}(\mathbf{r} \otimes \mathbf{r}) \otimes(\mathbf{r} \otimes \mathbf{r})+ \\
& +k^{f s} E_{t g}^{f s}(\mathbf{s} \otimes \mathbf{s}) \otimes(\mathbf{s} \otimes \mathbf{s})+ \\
& +4 G_{t g}^{f \tau}\left(k^{f r}+k^{f s}\right)(\mathbf{r} \otimes \mathbf{s})^{s} \otimes(\mathbf{r} \otimes \mathbf{s})^{s}
\end{aligned}
$$

in which $\mathbf{C}_{t g}^{m}=\partial \boldsymbol{\sigma}^{m} / \partial \boldsymbol{\varepsilon}, E_{t g}^{f(\bullet)}=\partial \sigma^{f} / \partial \varepsilon^{f(\bullet)}$ and $G_{t g}^{f \tau}=\partial \tau^{f} / \partial \gamma^{f}$ are the tangent operators of the corresponding constitutive relations.

Figure 2 illustrates the stress composition scheme for reinforced concrete, according to the presented approach.

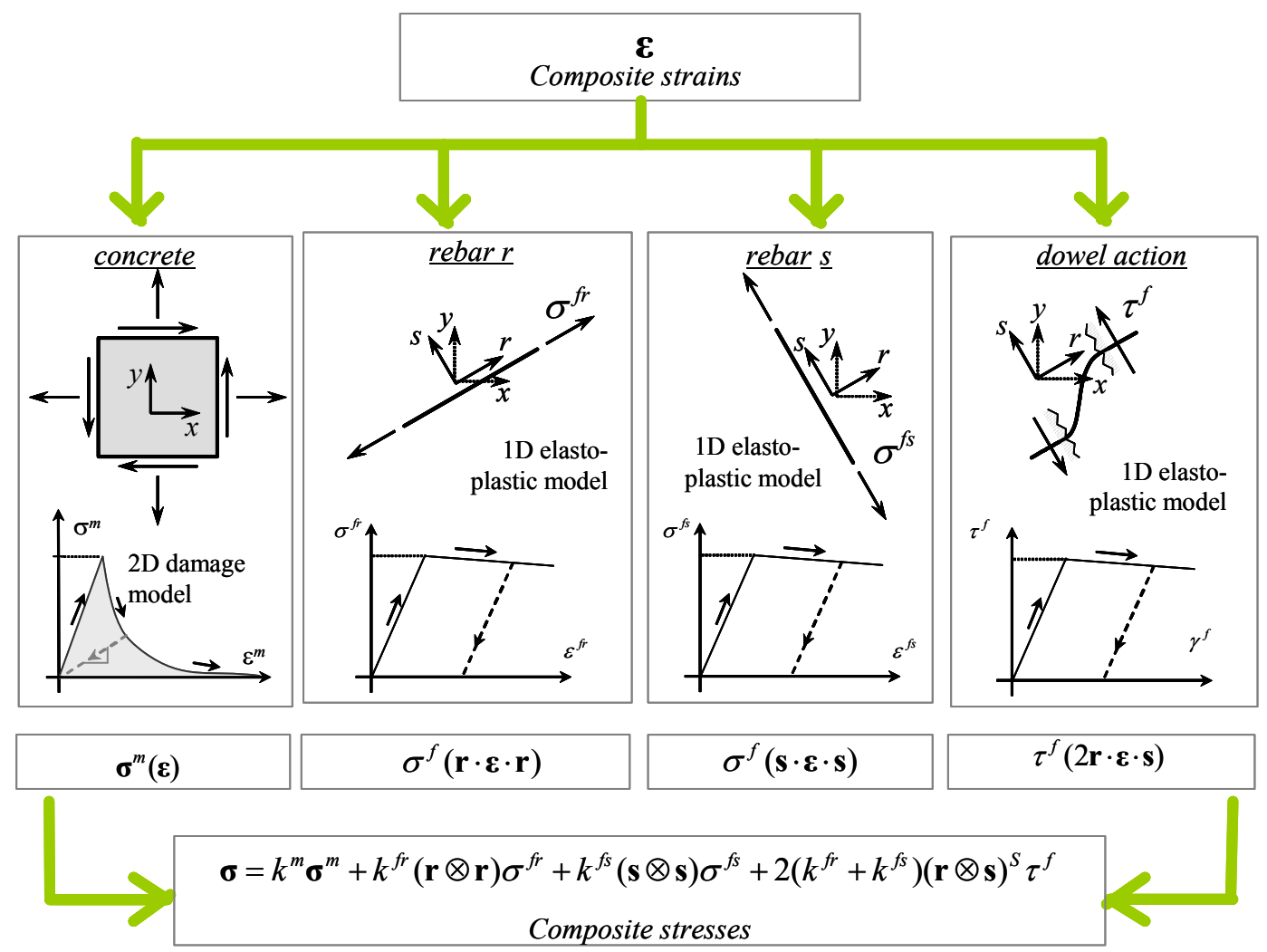

Figure 2. Stresses composition scheme for the composite material (reinforced concrete). 


\section{CONSTITUTIVE MODELS OF THE CONSTITUENTS}

General aspects of the constitutive modeling are the following:

- The matrix behavior is described by means of a non-symmetric tensile/compressive strength isotropic damage model, belonging to the family of damage models proposed by Simo and $\mathrm{Ju}$ [41], as presented in [24] and summarized in the Appendix.

- The rebar bond-slip effects are taken into account via a combination of the uniaxial elasto-plastic model, for the rebars, and a uniaxial slip dissipative model for the interface concrete-rebars, resulting in a unique constitutive model for the constituent slipping fiber.

- Additionally, the contribution of the rebars to the shear strength of the concrete (dowel action) is accounted by a combination of the previous slipping-fiber constitutive model with a uniaxial shear stress-strain dissipative model, resulting in a constitutive model for the constituent slipping-shear-resistant fiber.

The material instability and failure of the composite is determined via the discontinuous bifurcation analysis and the strong discontinuity analysis of the mixed model, providing: a) the time of crack onset and b) the propagation direction (exactly as if it were a single material).

\subsection{Matrix constitutive model: the distinct tensile-compression damage model}

The damage criterion is expressed in terms of the matrix strain field, $\varepsilon^{m}$, and the effective stress field $\bar{\sigma}^{m}$ :

$$
\begin{aligned}
& f\left(\boldsymbol{\varepsilon}^{m}, r\right)=\tau_{\varepsilon}-r \leq 0 \quad, \tau_{\varepsilon}=\alpha \sqrt{\bar{\sigma}^{m}:\left(\mathbf{C}^{m}\right)^{-1}: \bar{\sigma}^{m}} ; \\
& \overline{\boldsymbol{\sigma}}^{m}=\mathbf{C}^{m}: \varepsilon^{m} \quad ; \quad r(t)=\max _{s \in[0, t]}\left[r_{0}, \tau_{\varepsilon}\left(\boldsymbol{\varepsilon}^{m}(s)\right)\right] \quad ; \quad r_{0}=\frac{\sigma_{u}^{m}}{\sqrt{E^{m}}}
\end{aligned}
$$

where $\mathbf{C}^{m}$ is the Hooke's elastic constitutive tensor of the concrete, $r$ is the strain-like internal variable,$\sigma_{u}^{m}$ is the tensile strength, $E^{m}$ is the Young's modulus. In equation (4) the factor $\alpha$ is defined as:

$$
\alpha=\frac{\sum_{i=1}^{3}\left\langle\bar{\sigma}_{i}^{m}\right\rangle}{\sum_{i=1}^{3}\left|\bar{\sigma}_{i}^{m}\right|}\left(1-\frac{1}{n}\right)+\frac{1}{n}
$$

where $\bar{\sigma}_{i}^{m}$ is the $\mathrm{i}$-th principal effective stress, $\langle\bullet\rangle$ is the Mac-Auley bracket and $n$ is the ratio between the compression and tensile strengths (see Figure 3).

The stress rate $\dot{\sigma}^{m}$ is related to the strain rate $\dot{\varepsilon}^{m}$ as:

$$
\dot{\sigma}^{m}=\mathbf{C}_{t g}^{m}: \dot{\varepsilon}^{m}
$$

where the constitutive tangent tensor $\mathbf{C}_{t g}^{m}$ is given by:

$$
\mathbf{C}_{t g}^{m}=\frac{q}{r} \mathbf{C}^{m} ; \text { (unloading case) }
$$


$\mathbf{C}_{t g}^{m}=\frac{q}{r} \mathbf{C}^{m}-\left(\frac{q-H^{m} r}{r^{3}}\right) \cdot\left[\frac{r^{2}}{\alpha}\left(\overline{\boldsymbol{\sigma}}^{m} \otimes \mathbf{A}\right)+\alpha^{2}\left(\overline{\boldsymbol{\sigma}}^{m} \otimes \overline{\boldsymbol{\sigma}}^{m}\right)\right] ;$ (loading case)

$\mathbf{A}=\mathbf{C}^{m}: \partial_{\bar{\sigma}} \alpha$

where $q$ is the stress-like internal variable, dual of $r$. The evolution of the internal variables are related by the softening law $\dot{q}(t)=H^{m}(t) \dot{r}(t)$ with initial values $\left.r\right|_{t=0}=\left.q\right|_{t=0}=r_{0}=\sigma_{u}^{m} / \sqrt{E^{m}}$, where $H^{m}<0$ is the continuum softening modulus (see Appendix for additional information).

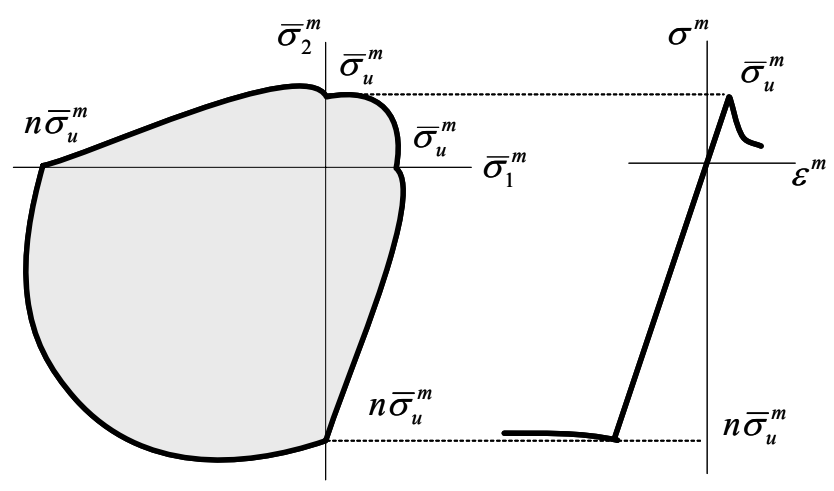

(a)

(b)

Figure 3 . Damage model with distinct tensile and compressive strengths: (a) Initial elastic domain in the stress space, (b) onedimensional stress-strain response.

\subsection{Constitutive model of the reinforcement bars (slipping-shear-resistant fiber model)}

\subsubsection{Uniaxial elasto-plastic model}

The axial behavior of the steel bars is described by means of a one-dimensional elastoplastic model, endowed with a linear softening law, defined by the following equations [40]:

$$
\left.\begin{array}{ll}
\sigma^{f}=E^{f}\left(\varepsilon^{f}-\varepsilon_{p}^{f}\right) & \text { (constitutive relation) } \\
\dot{\varepsilon}_{p}^{f}=\lambda^{f} \operatorname{sign}\left(\sigma^{f}\right) ; \dot{\alpha}^{f}=\lambda^{f} & \text { (internal variables evolution) } \\
f^{f}\left(\sigma^{f}, \alpha^{f}\right)=\left|\sigma^{f}\right|-\left(\sigma_{y}^{f}+q^{f}\right) & \text { (yield function) } \\
\dot{q}^{f}=H^{f} \dot{\alpha}^{f} & \text { (softening law) } \\
f^{f} \leq 0 ; \lambda^{f} \geq 0 ; \lambda^{f} f^{f}=0 \\
\lambda^{f} \dot{f}^{f}=0\left(f^{f}=0\right)
\end{array}\right\} \quad \begin{aligned}
& \text { (loading/unloading and } \\
& \text { persistency conditions) }
\end{aligned}
$$


where $\varepsilon_{p}^{f}$ is the plastic strain, $\alpha^{f}$ is the strain-like internal variable, conjugate of the stress-like variable $q^{f}, \lambda^{f}$ is the plastic multiplier, $\sigma_{y}^{f}$ is the yield stress, $E^{f}$ is the elastic modulus and $H^{f}$ is the hardening/softening modulus (supra index $f$ refers to fiber $r$ or $s$ ).

The rate form of the constitutive relation between the fiber axial stress, $\sigma^{f}$, and axial strain, $\varepsilon^{f}$, can be written as:

$\dot{\sigma}^{f}=E_{t g}^{f} \dot{\varepsilon}^{f}$

where the tangent modulus $E_{t g}^{f}$ is expressed in terms of $E^{f}$ and $H^{f}$, depending on the loading or unloading situation, i.e.:

$E_{t g}^{f}=\left\{\begin{array}{cc}E^{f} & \text { (elastic/ unloading) } \\ \frac{E^{f} H^{f}}{E^{f}+H^{f}} & \text { (loading) }\end{array}\right.$

Figure 5.c depicts the typical stress-strain curve obtained with this model.

\subsubsection{Axial resisting + bond-slip model (slipping-fiber model)}

To take into account the sliding and debonding mechanisms of the fiber-matrix interface, the fiber strain $\varepsilon^{f}$ is assumed as an additive composition of two parts: one due to the intrinsic fiber mechanical deformation, $\varepsilon^{d}$, and another associated with the interface sliding, $\varepsilon^{i}$, such that:

$\varepsilon^{f}=\varepsilon^{d}+\varepsilon^{i}$

Assuming a two-component serial system constituted by the fiber and the interface, as shown in Figure 4, the corresponding slipping-fiber stress $\sigma^{f}$ is identical to the stress of each component:

$\sigma^{f}=\sigma^{d}=\sigma^{i}$

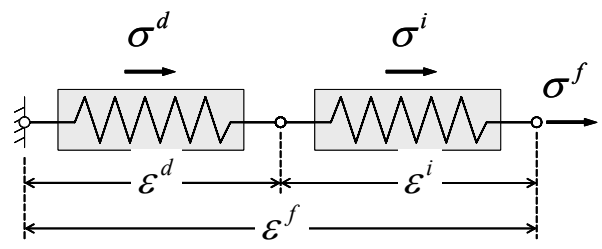

Figure 4. Slipping-fiber model.

Both stress-strain relations ( $\sigma-\varepsilon)$, describing the interface sliding mechanism and the fiber mechanical deformation, are also assumed to follow a one-dimensional elastoplastic model, as shown in Figure 5a-b. In this case, the resulting slipping-fiber constitutive model is still governed by equations (9)-(13), but with material parameters defined from the composition of both models, i.e:

$$
\begin{aligned}
& E^{f}=\frac{1}{1 / E^{d}+1 / E^{i}} \\
& \sigma_{y}^{f}=\min \left\lfloor\sigma_{y}^{d}, \sigma_{a d h}^{i}\right\rfloor
\end{aligned}
$$


$H^{f}=\left\{\begin{array}{lc}H^{d} & \text { if } \sigma_{y}^{d}<\sigma_{a d h}^{i} \\ H^{i} & \text { otherwise }\end{array}\right.$

in which $E^{d}$ and $\sigma_{y}^{d}$ are the steel Young's modulus and yield stress, respectively, $E^{i}$ is the interface elastic modulus and $\sigma_{a d h}^{i}$ is the interface bond limit stress, which is an upper bound for the fiber-matrix interface adherence (see Figure 5b).

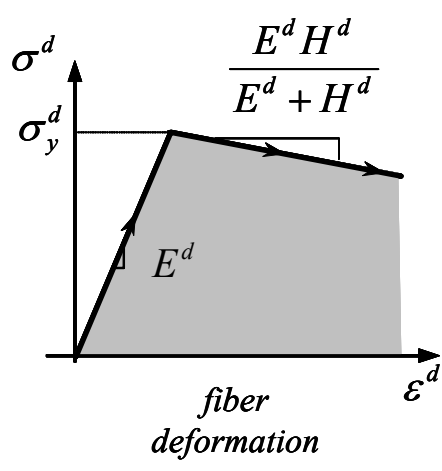

(a)

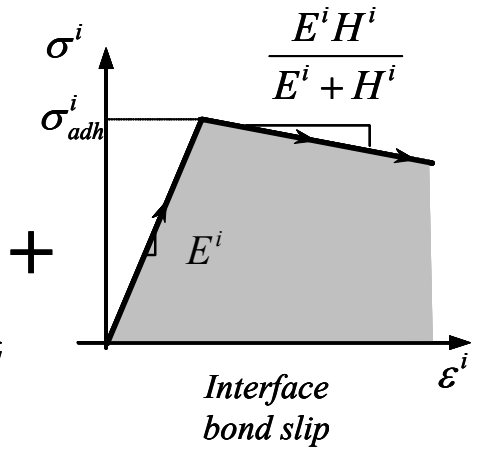

(b)

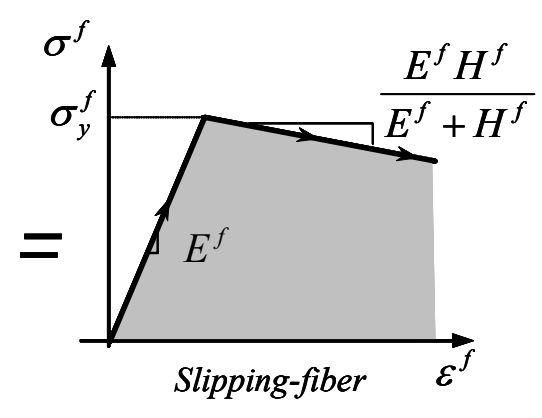

(c)

Figure 5. Slipping-fiber model composition.

Notice that if $E^{i} \rightarrow \infty$ and $\sigma_{a d h}^{i}>\sigma_{y}^{d}$, the system provides only the mechanical behavior of the fiber, reproducing a perfect connection between concrete and reinforcement bars.

\subsubsection{Properties characterization of the bond-slip model}

The parameters required for the bond/slip model characterization can be calibrated from the pull-out test, in which a bar embedded into a concrete core is subjected to a force applied at its free end, as shown in Figure 6. From the force-displacement curve obtained with this test (see Figure 6b), it is possible to evaluate the equivalent interface elastic modulus, bonding limit stress and softening modulus as:

$$
\begin{aligned}
& E^{i}=\left(\frac{P}{\delta}\right) \cdot\left(\frac{L_{s}}{A^{f}}\right) ; \sigma_{a d h}^{i}=\frac{P_{\max }}{A^{f}} ; \frac{E^{i} H^{i}}{E^{i}+H^{i}}=\lambda \cdot\left(\frac{L_{s}}{A^{f}}\right) \\
& A^{f}=\pi d^{2} / 4
\end{aligned}
$$

where $P$ and $\delta$ are, respectively, the values of the applied force and the corresponding measured displacement, $d$ is the diameter of the bar, $L_{s}$ is the embedded length and $\lambda$ is the observed softening slope. 


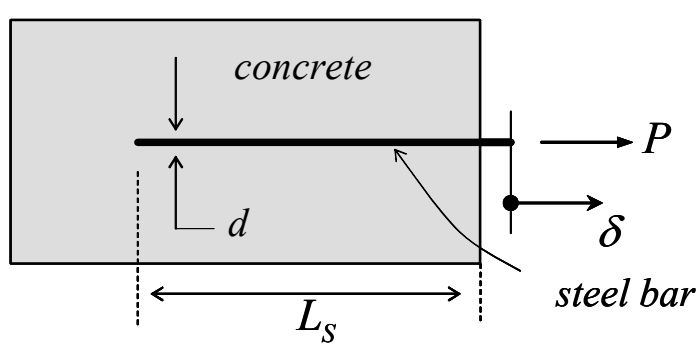

(a)

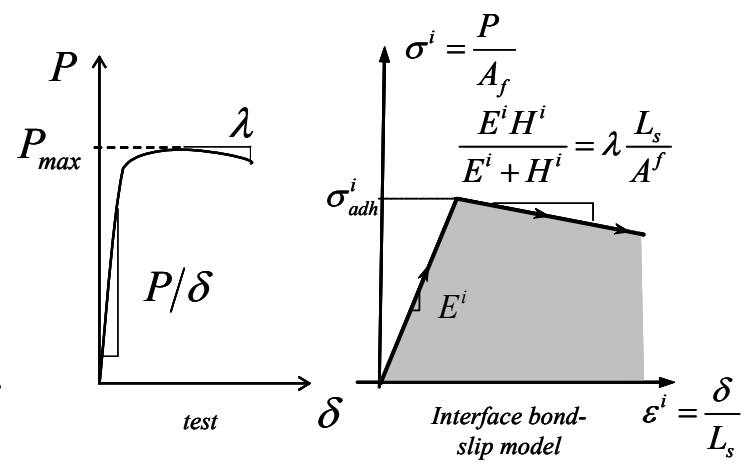

(b)

(c)

Figure 6. Pull out test: (a) test specimen, (b) experimental force-displacement curve, (c) bond-slip constitutive model.

\subsection{Constitutive model for rebars in shear mode (shear-resistant fiber). Dowel action model.}

The strengthening effect introduced by the rebar in reinforced concrete members loaded in shear has been widely studied (see reference [35] for instance) and several authors have included this effect into their numerical simulations $[4,13,36]$.

In a reinforced concrete member, when cracks open in mode II, the internal locking between particles (aggregate interlock) withstands some shear forces across the crack interfaces. The steel bars also introduce an important contribution to the shear strengthening effect, known as dowel action. This phenomenon is due to the following distinct mechanisms acting on the rebars embedded in the concrete:

(a) the beam bending mechanism, which is mainly activated in mixed-mode crack opening, where the crack interface separation $l$ (in mode I) is large enough to make relevant the bending of the bar [35]. To account for this effect one can assume a steel beam of length $l$ clamped at both ends, which are shifted, one with respect to the other, a displacement $\delta$, as shown in Figure 7a. Following the classical Timoshenko's beam theory, in the elastic range, the relative displacement $\delta$ determines the bar shear force, $V$, providing an equivalent shear elastic modulus, $G^{f}$, as follows:

$V=\frac{12 E^{d} I}{l^{3}} \delta=G^{f}\left(\frac{\delta}{l}\right) A^{f} \Rightarrow G^{f}=E^{d} \frac{12 I}{l^{2} A^{f}}$

where $I=\pi d^{4} / 64$ and $A^{f}=\pi d^{2} / 4$ are the momentum of inertia and the area of the cross section of a bar of diameter $d$.

The elastic regime of the beam is admissible whenever the maximum bending moment $M=V l / 2$ is smaller than the plastic moment $M_{Y}=\sigma_{Y}\left(d^{3} / 6\right)$, where $\sigma_{Y}$ is the steel yield stress. Therefore, the shear force corresponding to the elastic limit and the equivalent shear yield stress, $\tau_{y}^{f}$, can be determined as:

$V_{y}=\frac{4}{3 \pi} \cdot \frac{d}{l} \cdot A^{f} \sigma_{y}^{d}=\tau_{y}^{f} A^{f} \Rightarrow \tau_{y}^{f}=\frac{4}{3 \pi} \frac{d}{l} \sigma_{y}^{d}$ 
(b) the shear mechanism, which is dominant when the crack opens in mode II (or in a mixed-mode opening but with a dominant mode II), see Figure $7 \mathrm{~b}$. The interface shear strength is introduced by means of the bar shear force, which is given by:

$$
V=\frac{G^{f} A^{f}}{l} \delta \quad ; \quad G^{f}=\frac{E^{d}}{2(1+v)}
$$

where $v$ is the steel Poisson's ratio. Assuming the von Mises' yield criterion, the yield shear force $V_{y}$ for a pure shear stress state is:

$$
V_{y}=\frac{\sigma_{y}^{d}}{\sqrt{3}} A_{f}=\tau_{y}^{f} A_{f} \Rightarrow \tau_{y}^{f}=\frac{\sigma_{y}^{d}}{\sqrt{3}}
$$

In early stages of the inelastic regime, the cracks are distributed in the concrete matrix such that their opening remains small. Therefore, the second mechanism of the dowel action might describe more closely the bar strengthening effect in these stages. Since in this formulation the crack openings are assumed small, only that second mechanism is considered in section 6 .

Based on the previous considerations, the dowel action is modeled by means of a onedimensional shear stress-strain elasto-plastic constitutive model, similar to the previously mentioned one for the axial stress-strain. In this case, the $1 \mathrm{D}$ constitutive model relates the fiber shear stress, $\tau^{f}$, associated to the local coordinate system $(r, s)$ (see Figure 7), with the corresponding fiber shear stain, $\gamma^{f}$, by means of:

$\dot{\tau}^{f}=G_{t g}^{f \tau} \dot{\gamma}^{f}$

where the shear tangent modulus $G_{t g}^{f \tau}$ is given by:

$G_{t g}^{f \tau}=\left\{\begin{array}{cc}G^{f} & \text { (elastic/unloading) } \\ \frac{G^{f} H^{f \tau}}{G^{f}+H^{f \tau}} & \text { (loading) }\end{array}\right.$

The constitutive elastic shear modulus, $G^{f}$, is given either by Equation (22) or by Equation (24), depending on which aforementioned dowel action mechanisms is dominant. In the same way, the yield shear stress, $\tau_{y}^{f}$, is given either by Equation (23) or by Equation (25). The fiber shear hardening/softening modulus is commonly assumed as $H^{f \tau}=0$.

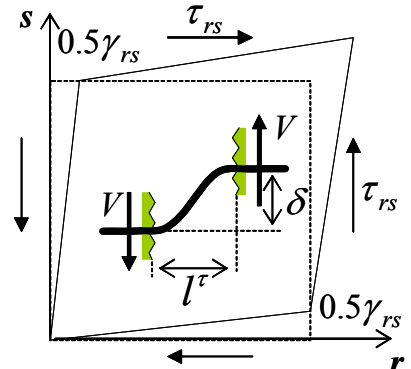

(a)

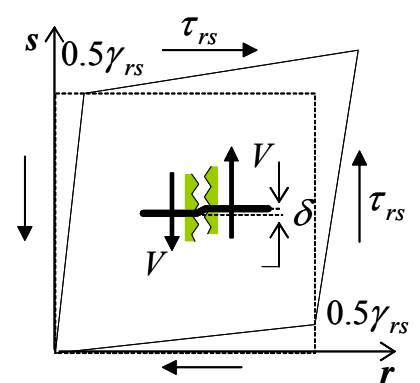

(b)

Figure 7. Dowel action: (a) bending of the rebar due to the crack opening, (b) direct shear of the rebar. 
The uncoupled axial stress-strain, $\sigma^{f}\left(\varepsilon^{f}\right)$, and shear stress-strain , $\tau^{f}\left(\gamma^{f}\right)$, constitutive relations, defined by the previous equations, constitute the slipping-shearresistant fiber model adopted for the rebars.

\section{THE CONTINUUM STRONG DISCONTINUITY APPROACH (CSDA) TO MATERIAL FAILURE}

Some ingredients of the CSDA, the framework where the present model is inserted, are presented in the following sections. Detailed aspects of the approach can be found in references [20, 21, 26, 29].

\subsection{Kinematical description of discontinuous displacement fields}

The CSDA aims at modeling discontinuous displacement/velocity fields (strong discontinuities) across a failure (discontinuity) line. Let $\Omega$ be a two-dimensional body displaying a strong discontinuity line $S$ with normal vector $\mathbf{n}$. The body is then splitted by this line into two parts: $\Omega^{+}$and $\Omega^{-}$(see Figure 1a). The velocity field at a point $\mathbf{x}$ and time $t$ is then expressed as:

$\dot{\mathbf{u}}(\mathbf{x}, t)=\dot{\overline{\mathbf{u}}}(\mathbf{x}, t)+M_{S}(\mathbf{x}) \cdot[[\dot{\mathbf{u}}]](\mathbf{x}, t)$

Where $\dot{\overline{\mathbf{u}}}$ and $[[\dot{\mathbf{u}}]]$ are, respectively, the rates of the regular (continuous) displacement and displacement jump; $M_{S}(\mathbf{x})$ is the unit jump function, defined by $M_{S}(\mathbf{x})=H_{S}(\mathbf{x})-\varphi(\mathbf{x})$, where $H_{S}(\mathbf{x})$ is the Heaviside's (step) function and $\varphi(\mathbf{x})$ an arbitrary continuous function with a small support in $\Omega^{h} \subset \Omega$ (see Figure 8):

$$
\begin{aligned}
& H_{S}(\mathbf{x})= \begin{cases}0 & \forall \mathbf{x} \in \Omega^{-} \\
1 & \forall \mathbf{x} \in \Omega^{+}\end{cases} \\
& \varphi(\mathbf{x})= \begin{cases}0 & \forall \mathbf{x} \in \Omega^{-} / \Omega^{h} \\
1 & \forall \mathbf{x} \in \Omega^{+} / \Omega^{h}\end{cases}
\end{aligned}
$$

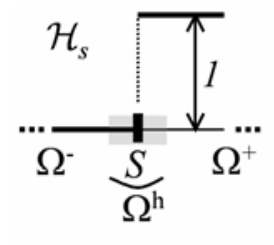

(a)

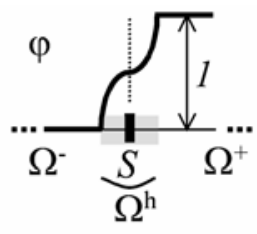

(b)

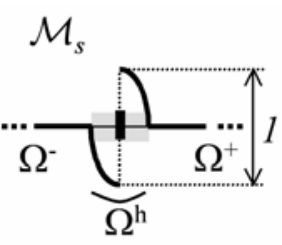

(c)

Figure 8. (a) Heaviside's function, (b) $\varphi$ function and (c) unit jump function.

The strain rate compatible with the velocity field of Equation (28) is then given by: 


$$
\dot{\boldsymbol{\varepsilon}}=\nabla^{S} \dot{\mathbf{u}}=\underbrace{\overbrace{\nabla^{S} \dot{\mathbf{u}}}^{\text {compatible }} \overbrace{-(\nabla \varphi \otimes[[\dot{\mathbf{u}}]])^{S}}^{\begin{array}{c}
\text { bounded } \\
\text { enhancement }
\end{array}}}_{\text {regular }}+\underbrace{\overbrace{\left(\delta_{S} \mathbf{n} \otimes[[\dot{\mathbf{u}}])^{S}\right.}^{\text {unbounded }} \text { enhancement }}_{\text {singular }}
$$

where $\delta_{S}$ stands for the line Dirac's delta function, whose regularized version, related to a regularizing volume $S^{k}$ of thickness $k$ (a regularization parameter as small as permitted by the machine precision) surrounding $S$, reads:

$$
\delta_{S}^{k}(\mathbf{x})=\lim _{k \rightarrow 0} \mu_{S}(\mathbf{x}) \frac{1}{k} ; \mu_{S}(\mathbf{x})= \begin{cases}1 & \mathbf{x} \in S^{k} \\ 0 & \mathbf{x} \notin S^{k}\end{cases}
$$

From Equations (30) and (31), the strain rate jump in $S^{k}$ is given by:

$$
[[\dot{\boldsymbol{\varepsilon}}]]=\dot{\boldsymbol{\varepsilon}}_{\Omega^{+} / S}-\dot{\boldsymbol{\varepsilon}}_{S}=\lim _{k \rightarrow 0}\left[\frac{1}{k}(\mathbf{n} \otimes[[\dot{\mathbf{u}}]])^{S}\right]
$$

\subsection{Softening modulus regularization}

In order to keep the bounded character of the stresses in front of the unbounded strains at the failure line (see equation (30)) the continuum softening modulus, $H^{(\bullet)}$, appearing in the constitutive model for all the constituents $(\bullet)$, has to be regularized as $H^{(\bullet)}=k \bar{H}^{(\bullet)}$, where $k$ is the regularization parameter used in equation (31) and $\bar{H}^{(\bullet)}$ is the so called intrinsic softening modulus, a material property to be determined in terms of the fracturing properties of the material [25]. For instance, for the damage (matrix) model for plain concrete, in section $3.1, \bar{H}^{m}$ is determined as;

$\bar{H}^{m}=-\frac{\left(\sigma_{u}^{m}\right)^{2}}{2 E^{m} G_{f}^{m}}$

where $G_{f}^{m}$ is the tensile fracture energy for plain concrete (to be determined by a direct or indirect tensile test). In the Appendix a detailed derivation of equation (33) is supplied.

As for the plastic (fiber) models for rebars, the value $\bar{H}^{f}$ is considered itself a material property to be determined from specific tests.

\subsection{Field equations}

The classical momentum equation and Neumann's boundary conditions can be written as:

$$
\begin{aligned}
& \nabla \cdot \dot{\boldsymbol{\sigma}}+\dot{\mathbf{b}}=\mathbf{0} ; \text { in } \Omega / S \\
& \dot{\boldsymbol{\sigma}} \cdot \boldsymbol{v}=\dot{\mathbf{t}}^{*} ; \text { in } \Gamma_{\sigma}
\end{aligned}
$$

where $\mathbf{b}$ stands for the body forces, $\mathbf{t}^{*}$ is the (prescribed) traction vector on the boundary, $\Gamma_{\sigma}$, and $v$ is the outward normal vector at that boundary.

In addition, the following boundary conditions should be prescribed at the discontinuity interface $S$ :

$$
\dot{\sigma}_{\Omega^{-}} \cdot \mathbf{n}-\dot{\sigma}_{\Omega^{+}} \cdot \mathbf{n}=\mathbf{0} \quad \text { in } S
$$


$\dot{\sigma}_{S} \cdot \mathbf{n}-\dot{\sigma}_{\Omega^{+}} \cdot \mathbf{n}=\mathbf{0} \quad$ in $S$

Equations (36) and (37) impose, respectively, the outer and inner traction continuity across the failure interface $S$.

The previous equations can be written in weak form by defining the test functions and the corresponding virtual strains:

$\boldsymbol{\gamma}=\underbrace{\nabla^{s} \overline{\boldsymbol{\eta}}}_{\text {compatible }}+\underbrace{-(\nabla \varphi \otimes \widetilde{\boldsymbol{\gamma}})^{S}+\delta_{S}(\tilde{\boldsymbol{\gamma}} \otimes \mathbf{n})^{S}}_{\text {enhanced }} ; \overline{\boldsymbol{\eta}} \in \overline{\mathrm{V}}_{u} \quad ; \quad \tilde{\boldsymbol{\gamma}} \in \tilde{\mathrm{V}}_{\varepsilon}$

where $\overline{\mathrm{V}}_{u}$ and $\widetilde{\mathrm{V}}_{\varepsilon}$ are the functional spaces for the admissible regular displacements, $\overline{\boldsymbol{\eta}}$, and element-wise constant displacement jumps, $\tilde{\gamma}$, respectively. Then, after insertions into the standard Virtual Work Principle, the weak form of equations (34)-(36) can be written as:

$$
\begin{aligned}
& \int_{\Omega} \dot{\sigma}: \nabla^{s} \overline{\boldsymbol{\eta}} d \Omega-\left[\int_{\Omega} \dot{\mathbf{b}} \overline{\boldsymbol{\eta}} d \Omega+\int_{\Gamma_{\sigma}} \dot{\mathbf{t}}^{*} \cdot \overline{\boldsymbol{\eta}} d \Gamma\right]=\mathbf{0} \quad \overline{\boldsymbol{\eta}} \in \overline{\mathrm{V}}_{u} \\
& \int_{\Omega^{e}} \dot{\boldsymbol{\sigma}} \cdot \nabla \varphi d \Omega-\int_{S^{e}} \dot{\boldsymbol{\sigma}} \cdot \mathbf{n} d S=\mathbf{0} \quad \forall e \in \mathrm{P}_{\Omega}
\end{aligned}
$$

where $\Omega^{e}$ is the domain of element $(e), S^{(e)}$ is the elemental counterpart of the discontinuity line, $S$, and $\mathrm{P}_{\Omega}$ stands for the set of elements intersected by $S$. It is worth nothing that the inner traction continuity condition (37) is introduced in weak form via equation (40). Additional information can be found in reference [29].

\section{ONSET OF FAILURE: MATERIAL BIFURCATION ANALYSIS}

Material bifurcation (localization) analysis introduces some specific features for the considered composite material with respect to the classical homogeneous case. Indeed, in this case, the localization direction and onset time depend on the mechanical properties of all constituents and not only on the matrix ones.

Considering a discontinuity line $S$ with normal vector $\mathbf{n}$, the rate form of the traction continuity condition reads:

$[[\dot{\boldsymbol{\sigma}} \cdot \mathbf{n}]]=[[\dot{\boldsymbol{\sigma}}]] \cdot \mathbf{n}=\dot{\sigma}_{\Omega+} \cdot \mathbf{n}-\dot{\boldsymbol{\sigma}}_{\Omega_{-}} \cdot \mathbf{n}=\mathbf{0}$

By considering the existence of solutions of equation (41) involving discontinuous deformations fields, $\dot{\boldsymbol{\varepsilon}}=\dot{\overline{\boldsymbol{\varepsilon}}}+\mu_{S}(\dot{\boldsymbol{\beta}} \otimes \mathbf{n})^{S}$, from equations (2) and (41) it is possible to obtain the classical localization condition:

$\left[\mathbf{n} \cdot \mathbf{C}_{t g} \cdot \mathbf{n}\right] \cdot \dot{\boldsymbol{\beta}}=\mathbf{Q}_{l o c}(t, \mathbf{n}) \cdot \dot{\boldsymbol{\beta}}=\mathbf{0}$

where $\dot{\boldsymbol{\beta}} \neq \mathbf{0}$ is a sufficient requirement for the existence of a localized strain discontinuity mode. Localization time, $t_{l o c}$, and localization direction, $\mathbf{n}_{l o c}$, can be determined by means of the singularity of the localization tensor $\mathbf{Q}_{l o c}$, corresponding to the tangent constitutive tensor of the composite material:

$\operatorname{det}\left(\mathbf{Q}_{l o c}\left(t_{l o c}, \mathbf{n}_{l o c}\right)\right)=0$

Inserting $\mathbf{C}_{t g}$ of Equation (3) into Equation (42), the localization tensor can be written as: 


$$
\begin{aligned}
\mathbf{Q}_{l o c}= & k^{m} \mathbf{Q}_{l o c}^{m}+k^{f r} E_{t g}^{f}(\mathbf{n} \cdot \mathbf{r})^{2}(\mathbf{r} \otimes \mathbf{r})+ \\
& +k^{f s} E_{t g}^{f}(\mathbf{n} \cdot \mathbf{s})^{2}(\mathbf{s} \otimes \mathbf{s})+4\left(k^{f r}+k^{f s}\right) G_{t g}^{f}\left[\mathbf{n} \cdot(\mathbf{r} \otimes \mathbf{s})^{s} \otimes(\mathbf{r} \otimes \mathbf{s})^{s} \cdot \mathbf{n}\right]
\end{aligned}
$$

in which $\mathbf{Q}_{l o c}^{m}$ is the matrix localization tensor for the isotropic damage model described in Section 3.1:

$\mathbf{Q}_{l o c}^{m}=\mathbf{n} \cdot \mathbf{C}_{t g}^{m} \cdot \mathbf{n}=\frac{q}{r}\left(\mathbf{n} \cdot \mathbf{C}^{m} \cdot \mathbf{n}\right)-\left(\frac{q-H^{m} r}{r^{3}}\right)\left(\frac{r^{2}}{\alpha}\left(\bar{\tau}^{m} \otimes \bar{\tau}^{A}\right)+\alpha^{2}\left(\bar{\tau}^{m} \otimes \bar{\tau}^{m}\right)\right)$

with $\bar{\tau}^{m}=\mathbf{n} \cdot \bar{\sigma}^{m}$ and $\bar{\tau}^{A}=\mathbf{n} \cdot \mathbf{A}$.

For the considered 2D problems, the localization time, $t_{l o c}$, and the discontinuity direction, $\mathbf{n}_{l o c}$, are evaluated by means of a numerical procedure to $\operatorname{minimize} \operatorname{det}\left(\mathbf{Q}_{l o c}\right)$ with respect to the localization angle, $\theta(\mathbf{n}=[\cos \theta, \sin \theta])$, formed by $\mathbf{n}$ and the first principal direction. Figure 9 shows the typical evolution of $\operatorname{det}\left[\mathbf{Q}_{\text {loc }}(\theta(\mathbf{n}))\right]$ up to reaching the localization condition. It can be observed that, unlike for homogeneous material, which may exhibit two different localization angles as solution of Equation (43), the localization condition for the composite material provides, due to the anisotropy supplied by the rebars, a single value for the localization angle $\theta_{l o c}$.
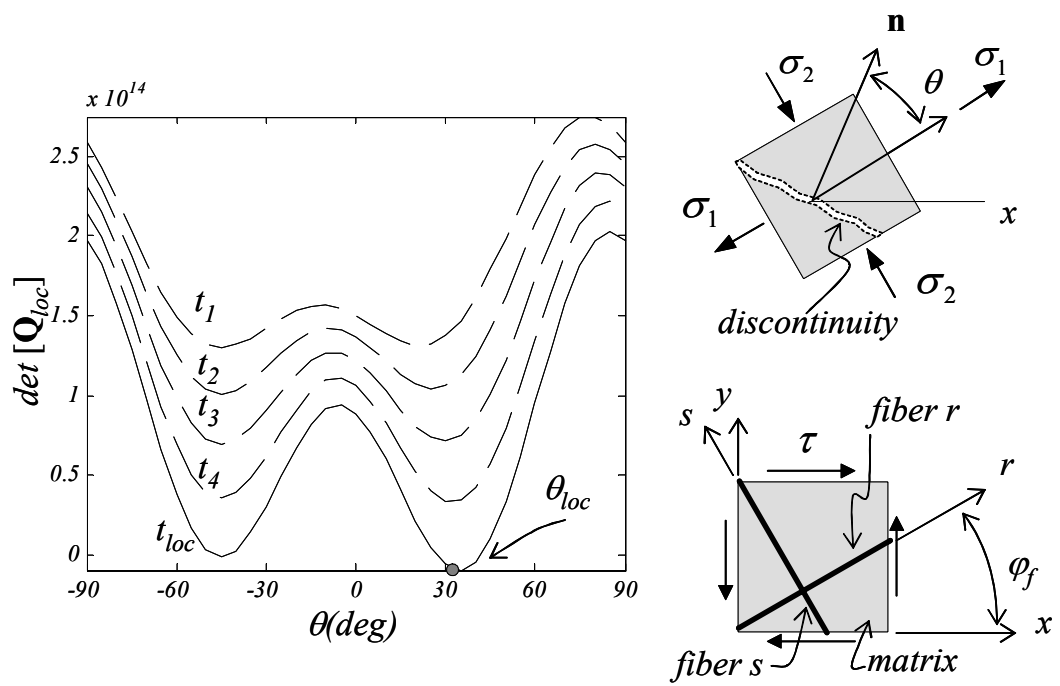

Figure 9. Localization analysis of a composite material under pure shear. Material properties: concrete: $E^{m}=20.0 \mathrm{GPa}, v=0.2, G_{f}^{m}=100 \mathrm{~N} / \mathrm{m}$, $\sigma_{u}^{m}=2.00 \mathrm{MPa}, n=10$; steel: $E^{f}=200.0 \mathrm{GPa}, \varphi_{f}=30^{\circ}, k^{f^{r}}=k^{f_{s}}=0.01785$.

\section{SAMPLE TESTS}

In order to assess the ability of the proposed model to capture material failure phenomena in reinforced concrete members, some experimental tests have been reproduced.

For a better understanding of the presented numerical results, the following remarks have to be done:

- Developing (active) cracks can be identified via the inelastic loading (active) or the elastic unloading (inactive) status of the corresponding material points. 
Therefore, plots of the variable describing the inelastic loading of the elements identify developing crack patterns in the figures.

- Developed crack patterns can be identified from the iso-displacement contour lines. In fact, full development of a crack is due to a strain localization process, which translates into grouping of the displacement contours. Therefore, the concentration of these contours into a band of elements identifies a developed crack in the figures.

- Graphic descriptions of developed and developing crack patterns, via the aforementioned procedure, may not coincide. Typically, a distributed developing crack pattern will show a diffuse spot in terms of the loading variable and no displacement contour concentration. On the contrary, a fully developed crack will translate into a band of elements in loading status, along the crack path, surrounded by inactive elements in elastic unloading. In this case the crack path can also be identified by a concentration of the displacement contours.

\subsection{Reinforced concrete panel under tension}

The reinforced concrete panel subject to uniaxial tension reported by Ouyang and coworkers $[32,33]$ has been modeled. The test consists of a panel with dimensions 686 $\mathrm{mm} \times 127 \mathrm{~mm}$ and $50.8 \mathrm{~mm}$ of thickness, reinforced with three steel bars of diameter $9.5 \mathrm{~mm}$. A weaker zone was introduced by means of a notch placed at its middle section, as shown in Figure 10a.

The concrete mechanical properties are: Young's modulus: $E^{m}=27.35 \mathrm{GPa}$, Poisson's ratio: $v^{m}=0.2$, fracture energy: $G_{f}^{m}=100 . \mathrm{N} / \mathrm{m}$ and tensile strength: $\sigma_{u}^{m}=3.19 \mathrm{MPa}$. The steel rebars correspond to $3.3 \%$ of the total panel volume. The steel elastic modulus and yield stress were $E^{d}=191.6 \mathrm{GPa}$ and $\sigma_{y}^{d}=508.0 \mathrm{MPa}$, respectively.

The debonding phenomenon between matrix and fibers has been characterized by means of the pull-out experiment reported by Naaman et al.[18]. Since the slope of the load-displacements curve $(P / \delta)$ is into the range [8.01 - 9.26] $\mathrm{kN} / \mathrm{mm}$, the pseudo-elastic modulus, $E^{i}$, evaluated with expression (21), is in the range [1111-1283] GPa and the resulting sliding fiber modulus, $E^{f}$, evaluated with expression (18), corresponds to 85$87 \%$ of the steel Young's modulus. The peak bond stress was evaluated via equation (21), yielding $\sigma_{a d h}^{i}=311.1 \mathrm{MPa}$.
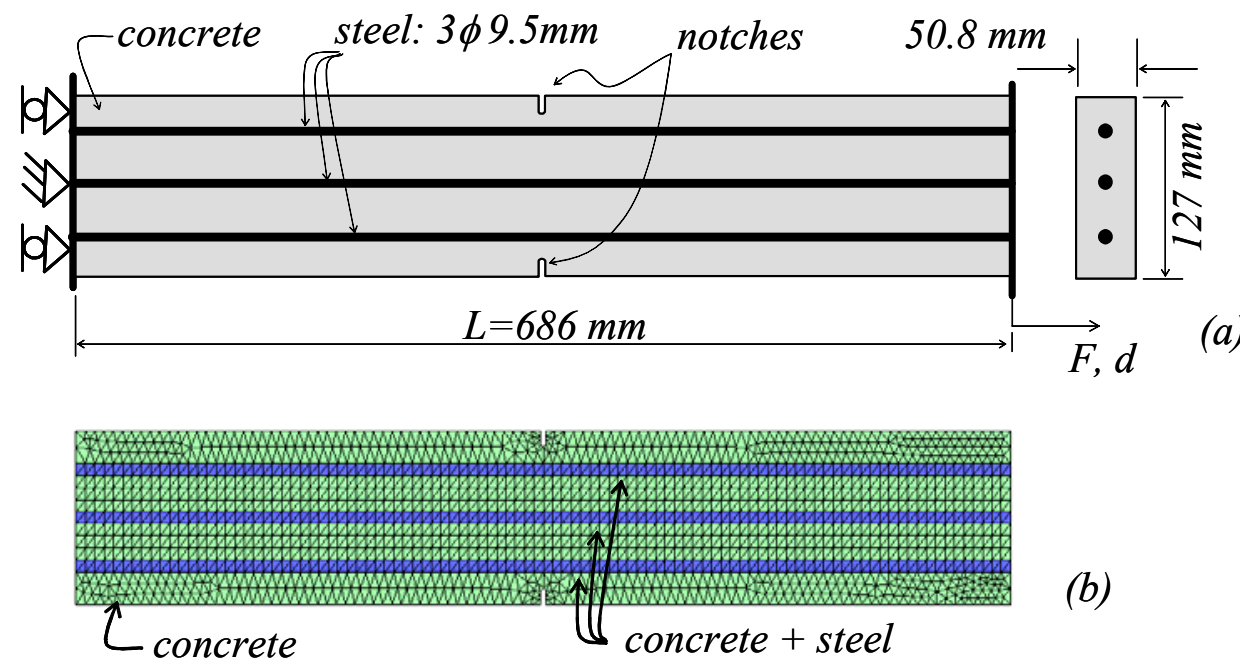

(b)

Figure 10. Reinforced concrete panel under uniaxial tension: (a) geometrical description ,

(b) finite element mesh 
The finite element mesh is made of linear triangular elements arranged as follows: $a$ ) three bands (one-element width) of composite material with uniformly distributed rebars (concrete + steel in Figure 10b), modeled as described in section $3 ; b$ ) a plain concrete material in the rest of the panel (concrete in Figure 10b), modeled by the damage model described in subsection 3.1. Notice that the rebars are macroscopically modeled by assuming uniform distribution across the elements in the concrete + steel zone.
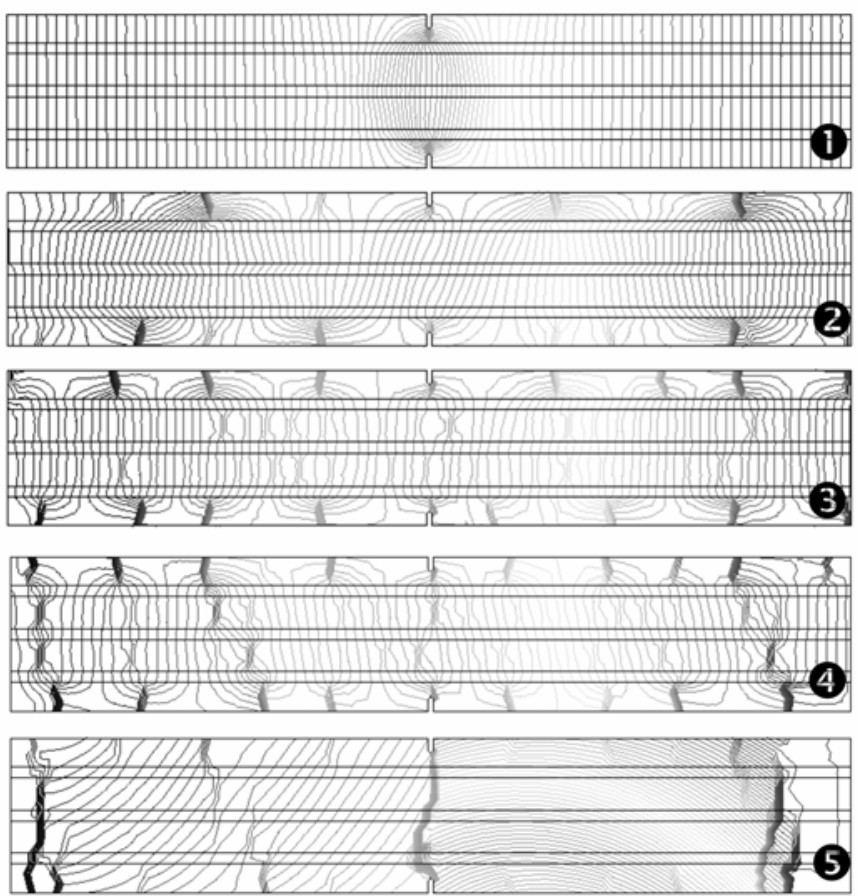

Figure 11. Reinforced concrete panel under uniaxial tension. Developed crack patterns identified via the norm of displacements contours.

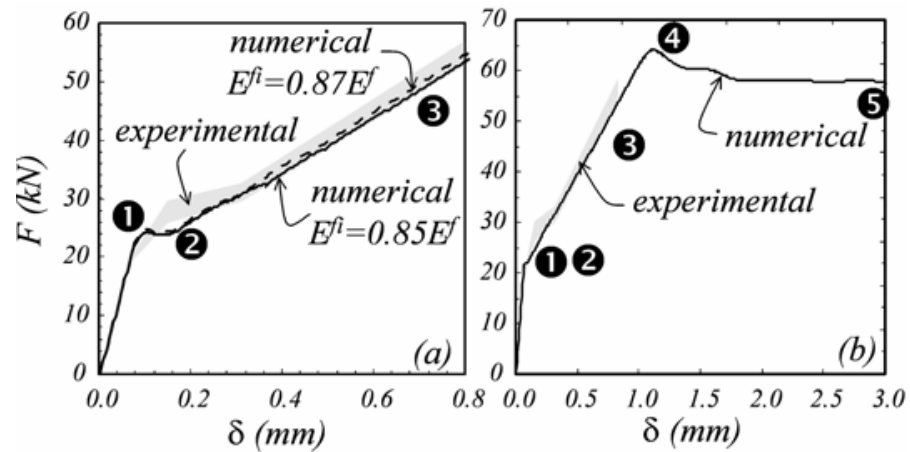

Figure 12. Reinforced concrete panel under uniaxial tension. Experimental and numerical force-displacement results for different stages of the loading process.

The iso-displacement contours, shown in Figure 11, describe the developed crack patterns at different stages of the loading process illustrated in Figure 12. In stage 1, the behavior corresponds to the elastic branch in Figure 12, in which no fully developed crack can be observed. Stages 2 and 3 correspond to the development of a distributed crack pattern, equi-spaced, at the upper and lower zones of the panel. Notice that this reproduces the well known phenomenon of the saturation distance [15], which is naturally captured in this approach without introducing any ad-hoc parameter. At these 
stages, crack penetration towards the panel core is arrested by the reinforcement, which is still in elastic (full bonding) regime. The corresponding loss of structural stiffness can be noticed in Figure 12, agreeing very well with the experimental results (in grey). Although no experimental results after this stage were reported, the numerical simulation could be taken further on to steps 4 and 5, beyond the onset of the rebars debonding. As it can be seen in Figure 11, the slipping-fiber failure of the rebars substantially modifies the crack pattern: some cracks get arrested while others develop penetrating the panel core, leading to the structural failure mode of the panel. The failure is characterized by the complete loss of stiffness at the critical stage 4 with a subsequent softening branch in Figure 12.

\subsection{Three-point reinforced concrete beam}

To demonstrate the ability of the model to properly describe the fiber-matrix slipping mechanism, the numerical simulation of the three point beam tested by Ruiz et al. [38] is presented. The experimental setting and the geometrical dimensions are shown in Figure 13. Four steel bars, of diameter $d=2.5 \mathrm{~mm}$ each, reinforce the concrete beam with a percentage of steel/concrete (in volume) equal to $0.13 \%$. Two types of material are assumed in the finite element model shown in Figure 13d: the elements in light grey represent the plain concrete, which is assumed a homogenous material described by the damage model of section 3. The elements in deep grey, forming a band of width $h_{e}=4 d$, represent a composite material constituted by concrete and rebars. With the adopted dimensions, the composite material has a volume fraction $k^{f}=0.0393$. The properties of concrete and steel bars are given in Table I.

\begin{tabular}{|c|c|c|c|c|}
\hline \multicolumn{5}{|c|}{ Table I: material parameters of the slightly reinforced concrete beam } \\
\hline \multicolumn{5}{|c|}{ Concrete } \\
\hline $\begin{array}{l}\text { Young's modulus } \\
E^{m}=29 \cdot[G P a]\end{array}$ & $\begin{array}{l}\text { Poisson's ratio } \\
v^{m}=0.2\end{array}$ & $\begin{array}{l}\text { Compression strength } \\
\sigma_{u(c)}^{m}=38.0[\mathrm{MPa}]\end{array}$ & $\begin{array}{l}\text { Tensile strength } \\
\sigma_{u}^{m}=3.8[M P a]\end{array}$ & $\begin{array}{l}\text { Fracture energy } \\
G_{f}^{m}=62.5[\mathrm{~N} / \mathrm{m}]\end{array}$ \\
\hline \multicolumn{5}{|c|}{ Steel bars } \\
\hline \multicolumn{2}{|c|}{$\begin{array}{c}\text { Young's modulus } \\
E^{d}=162 .[G P a]\end{array}$} & $\begin{array}{c}\text { Softening modulus } \\
H^{d}=0 .[G P a]\end{array}$ & \multicolumn{2}{|c|}{$\begin{array}{c}\text { Yield stress: } \\
\sigma_{y}^{d}=587 .[\mathrm{MPa}]\end{array}$} \\
\hline
\end{tabular}

The characterization of the interface model is based on results of the pull-out test reported in reference $[18,38]$, rendering $E^{i}=611.1 \mathrm{GPa}$ and $\sigma_{a d h}^{i}=570.4 \mathrm{MPa}$.

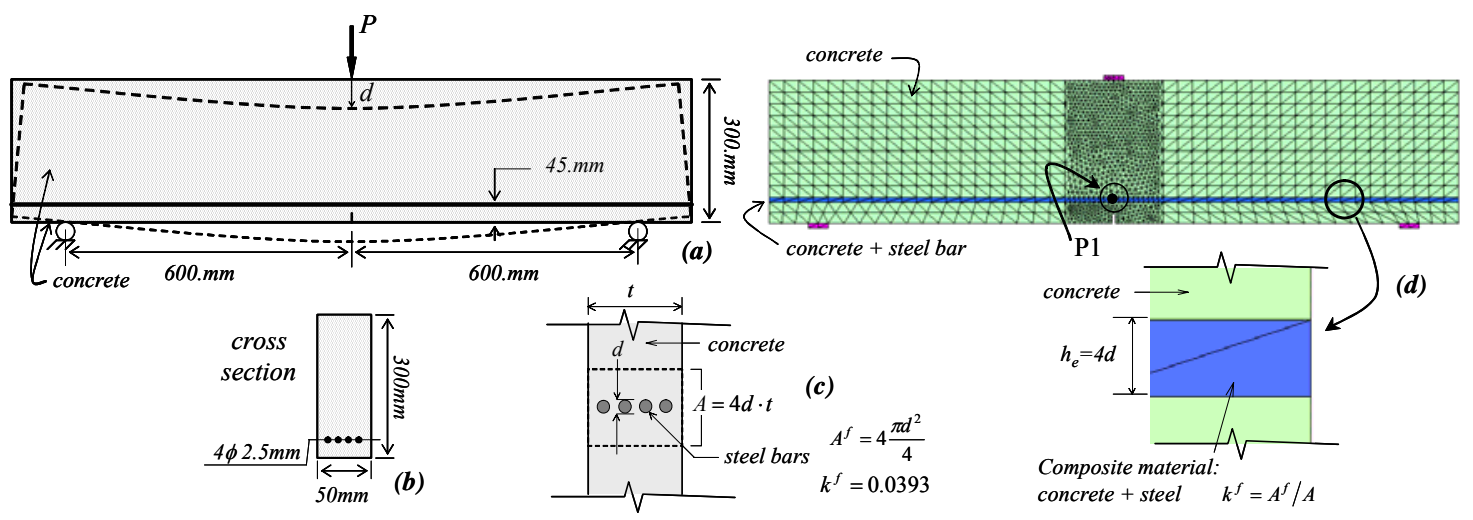

Figure 13. Lightly reinforced concrete beam. 
Figure 14a shows the experimental and predicted structural responses, as well as the six stages chosen for analysis. Figure 14b-d shows the stress evolution for each constituent of the mixture at the material point P1 indicated in Figure 13d. As it can be seen, the ultimate loading capacity (stage 2) corresponds to the matrix failure in b. The early post-critical response, between stages 2 and 4, corresponds to the degradation process of the concrete and the elastic behavior of the steel bars. In consequence, the slope of the structural curve in this branch depends strongly on the steel participation. After stage 4, the structural capacity is slightly recovered due to the predominance of the rebars (elastic) contribution. This recovering ceases around stage 5, in which the system barsinterfaces (slipping-fiber) undergoes plastic regime. Notice the close correspondence between the experimental and numerical responses.
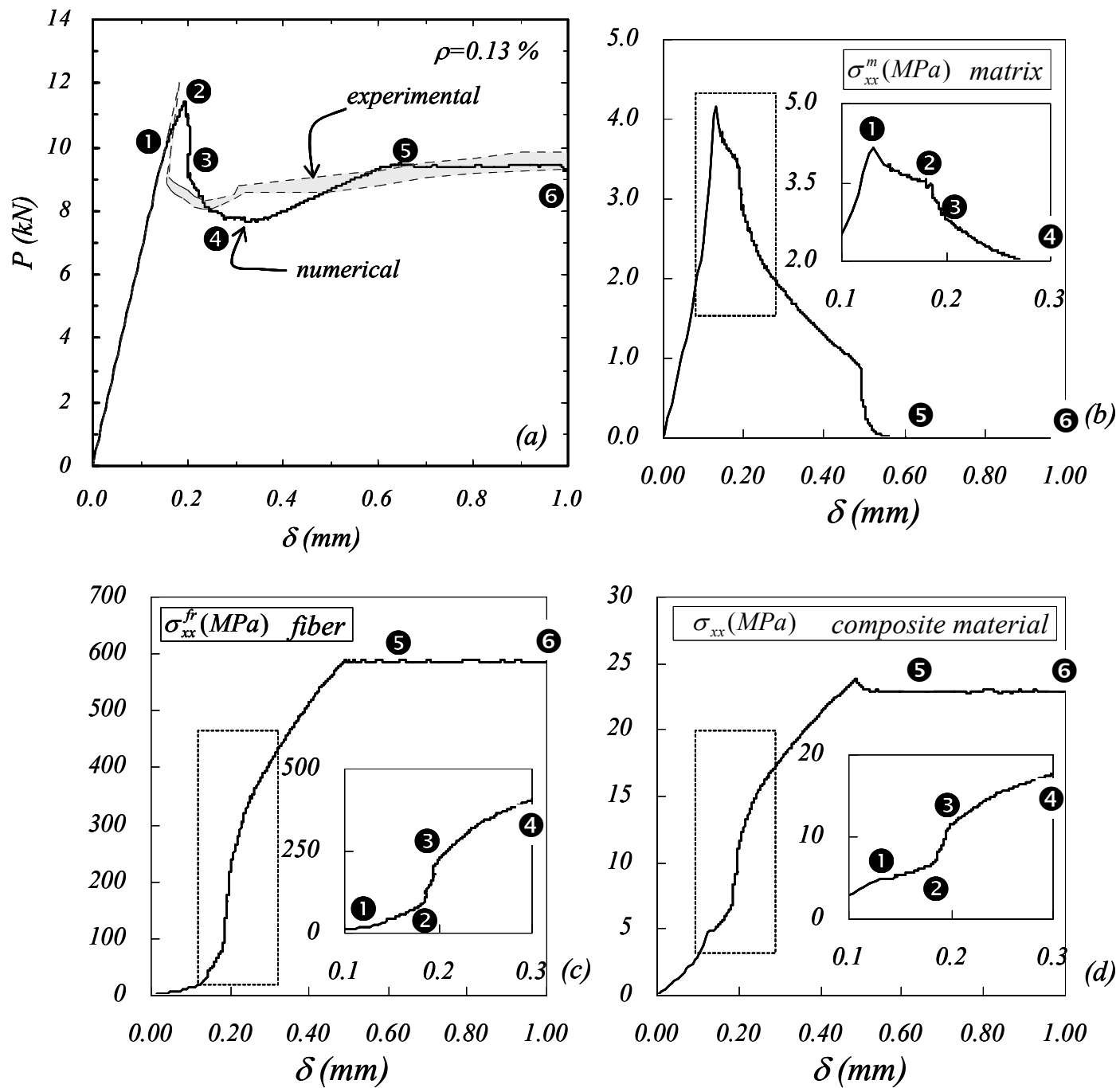

Figure 14. Slightly reinforced concrete beam: a) load vs. vertical displacement curve; b) matrix stress $\sigma_{x x}^{m}$ in point $\mathrm{P} 1$; c) fiber stress $\sigma^{f}$ in point $\mathrm{P} 1$; d) composite stress $\sigma_{x x}$ in point $\mathrm{P} 1$;

Finally, Figure 15 illustrates the evolution of the localization process at the chosen loading stages. It can be observed the propagation of the main macro-crack as well as the development of an orthogonal micro-cracking pattern in the concrete surrounding the rebar. 


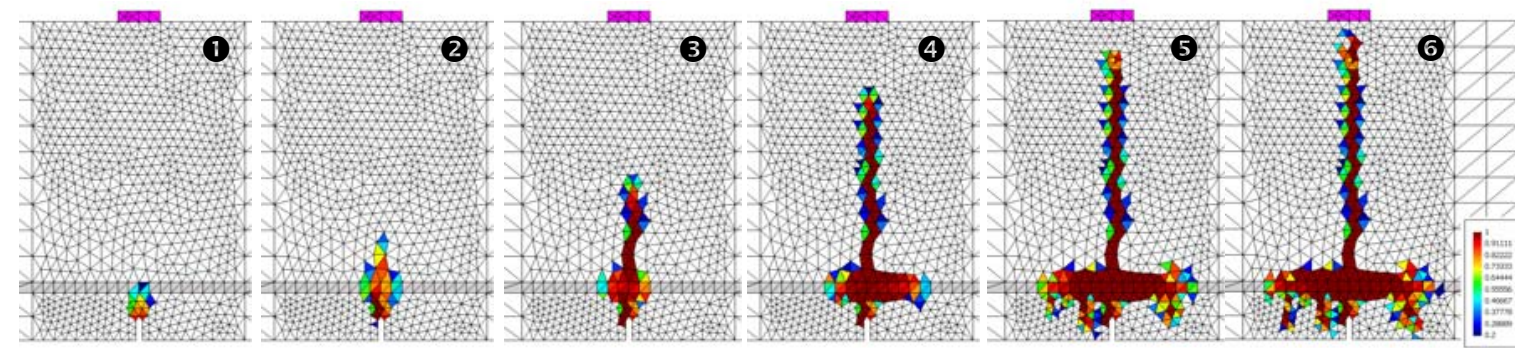

Figure 15. Slightly reinforced concrete beam: active crack pattern at different stages pf the processs

\subsection{Heavily reinforced beams}

The next example shows the ability of the proposed methodology to predict crack patterns in heavily reinforced beams. For this purpose, the set of reinforced beams reported by Leonhardt [14] is considered.

The experimental setting is shown in Figure 16a: a set of heavily reinforced concrete beams, with different aspect ratios is subjected to bending/shear under a couple of vertical forces, so that the central part of the beam is works under pure bending (no shear force) and the remaining works under a combined bending/shear action. Lower aspect ratios, $a / d$, correspond to dominant shear in the member, whereas dominant bending corresponds to high aspect ratios.

The reinforcement is constituted by two longitudinal steel bars of diameter $\phi=24 \mathrm{~mm}$, distributed as shown in Figure 16a. The assumed properties of the concrete and steel bar are shown in Table II.

\begin{tabular}{|l|l|l|l|l|}
\hline \multicolumn{6}{|c|}{ Table II: material parameters of the heavily reinforced concrete beam } \\
\hline \multicolumn{7}{|c|}{ Concrete } \\
\hline Young's modulus & Poisson's ratio & Compression strength & Tensile strength & Fracture energy \\
$E^{m}=20 .[G P a]$ & $v^{m}=0.2$ & $\sigma_{u(c)}^{m}=20 .[\mathrm{MPa}]$ & $\sigma_{u}^{m}=2 .[\mathrm{MPa}]$ & $G_{f}^{m}=60 .[\mathrm{N} / \mathrm{m}]$ \\
\hline \multicolumn{6}{|c|}{ Steel bars } \\
\hline \multicolumn{7}{|c|}{ Young's modulus } & Poisson's ratio & Softening modulus & Yield stress: \\
$E^{d}=200 .[G P a]$ & $v^{d}=0.2$ & $H^{d}=0 .[\mathrm{GPa}]$ & $\sigma_{y}^{d}=456 .[\mathrm{MPa}]$ \\
\hline
\end{tabular}

The interface fiber-matrix model parameters is evaluated from the experimental pull-out test of Naaman [18], rendering $E^{i}=1111.1 \mathrm{GPa}$ and $\sigma_{a d h}^{i}=311.1 \mathrm{MPa}$.

The dowel action is introduced by assuming $G^{f}=E^{d} / 2\left(1+v^{d}\right)=83.3 \mathrm{GPa}, \tau_{y}^{f}=$ $\sigma_{y}^{d} / \sqrt{3}=263.3 \mathrm{MPa}$ and perfect plasticity .

The finite element mesh is shown in Figure 16b, where, due to the symmetry shown by the experimental results, only one half of the beam has been modeled.
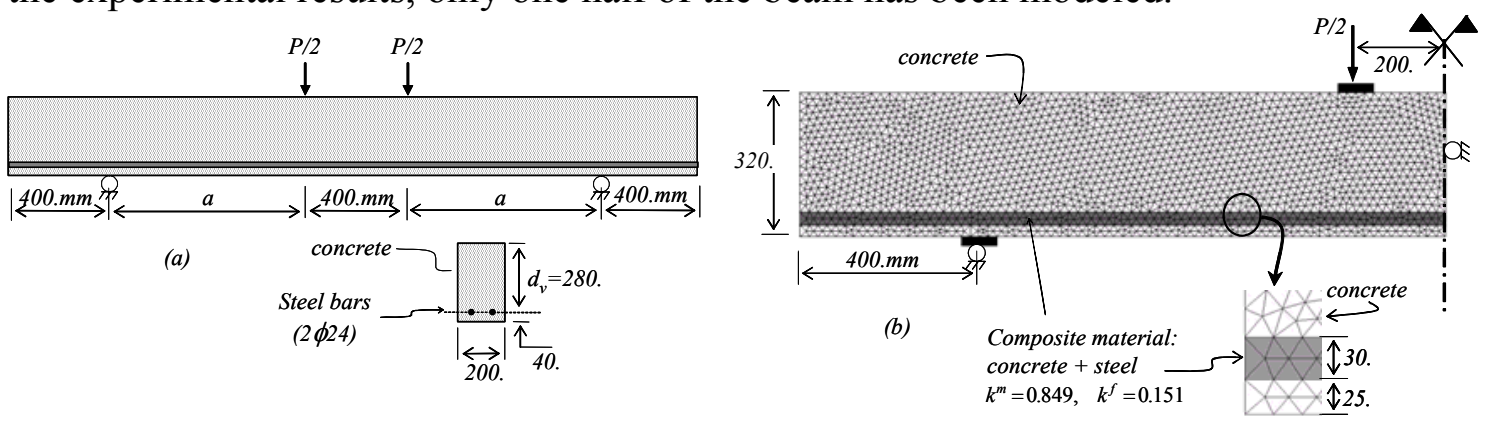

Figure 16. Heavily reinforced beams. 
The elements in light grey represent a homogeneous material with the properties of concrete in Table II. The zone in deep grey, of height equal to $30 . \mathrm{mm}$, represents a composite material with a volume fraction $k^{f}=0.151$. The fiber properties are those of the steel in Table II.

In Figure 17a the typical action-response curve obtained for one of the beams (aspect ratio: $a / d=3.0$ ) is presented. As it can be observed there, the totality of the curve (precritical and post-critical responses) can be obtained.

Before reaching the structural limit load, heavily reinforced members typically present multiple crack patterns propagating in a stable mode during a long period. In the postcritical regime, most of them become inactive and the collapse mechanism is characterized by one or few active cracks. Figure 18 illustrates the predicted evolution of the active cracks for the simulated beam, at the stages signaled in Figure 17a, which reproduces well the aforementioned phenomenon: at initial stages a multiple crack pattern develops from the center of the span to the end, and from the bottom to the top. In later stages, most of the vertical cracks arrest and a typical, inclined, shear/bending crack characterizes the final failure mode (see also Figure 17).
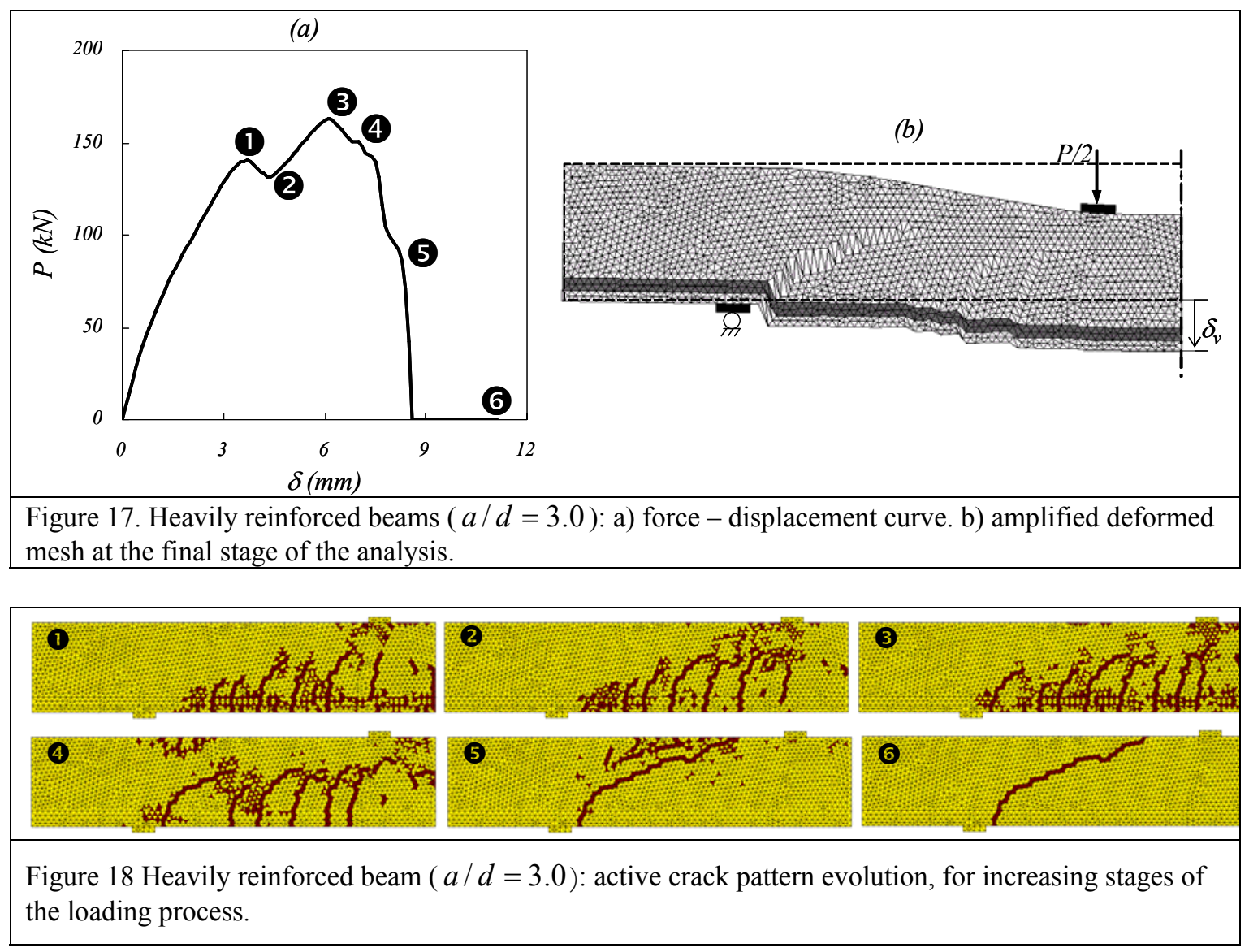
(a)

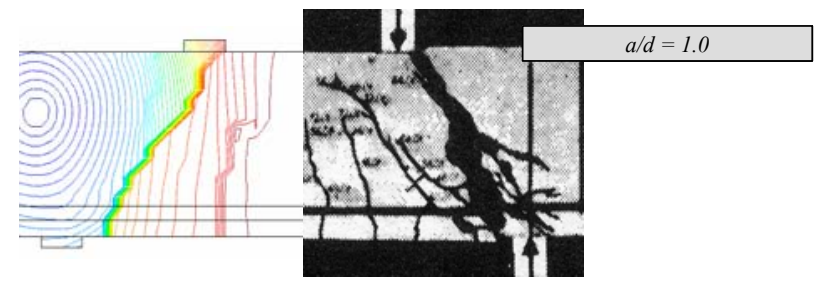

(b)

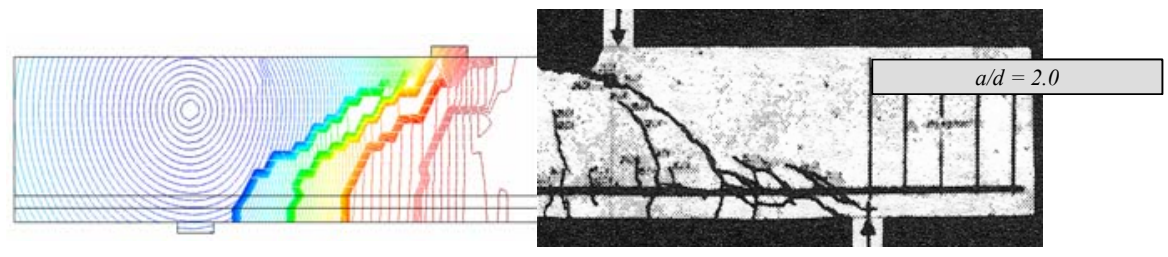

(c)
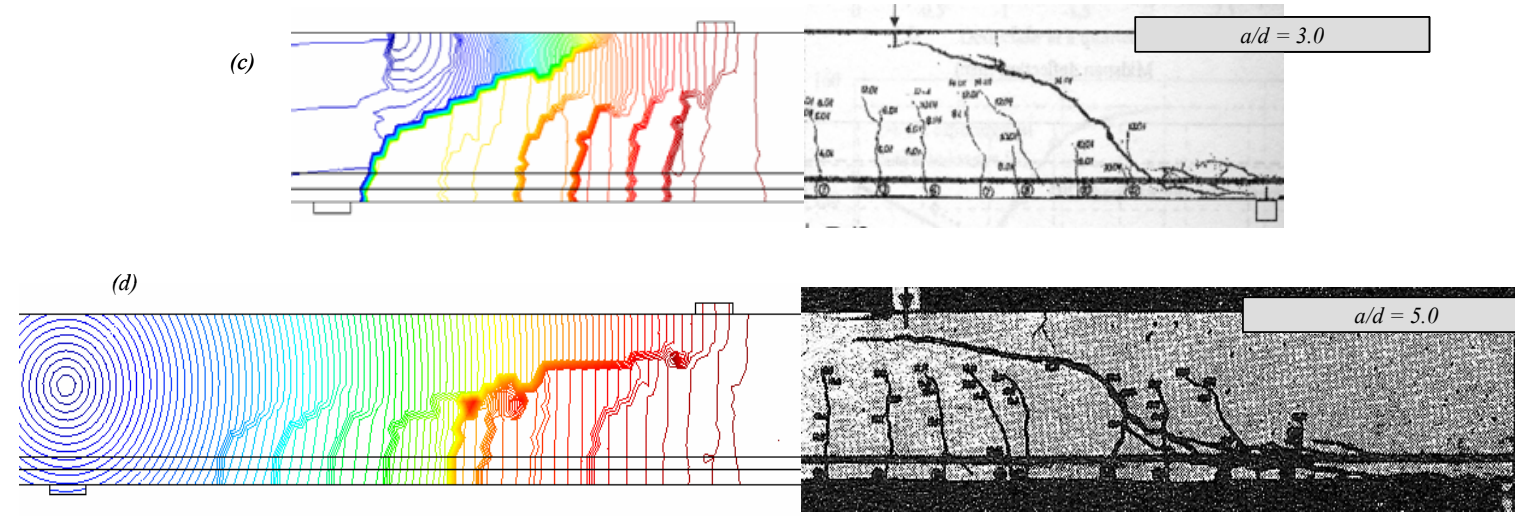

Figure 19. Heavily reinforced beams: comparison between numerical (indicated by concentration of the displacements contours) and experimental crack patterns for different aspect ratios.

Figure 19 shows the comparison between the numerically predicted and the experimentally obtained crack pattern for beams with different aspect ratios. The numerical crack patterns are illustrated by iso-displacement contours taken at the stage of analysis with largest number of active cracks. As it can be seen, calculated and observed crack patterns resemble very well in all cases.

\subsection{Reinforced concrete panel under pure shear loading}

In order to check the model ability to reproduce shear dominant stress states, the experimental test PV27 reported in Collins et al.[7] has been chosen. In this test, a reinforced concrete panel is loaded in pure shear, as illustrated in Figure 20a. The panel is $890 \mathrm{~mm}$ square by $70 \mathrm{~mm}$ thick, equally reinforced in two orthogonal directions, with a volume fraction of $1.785 \%$ each. 


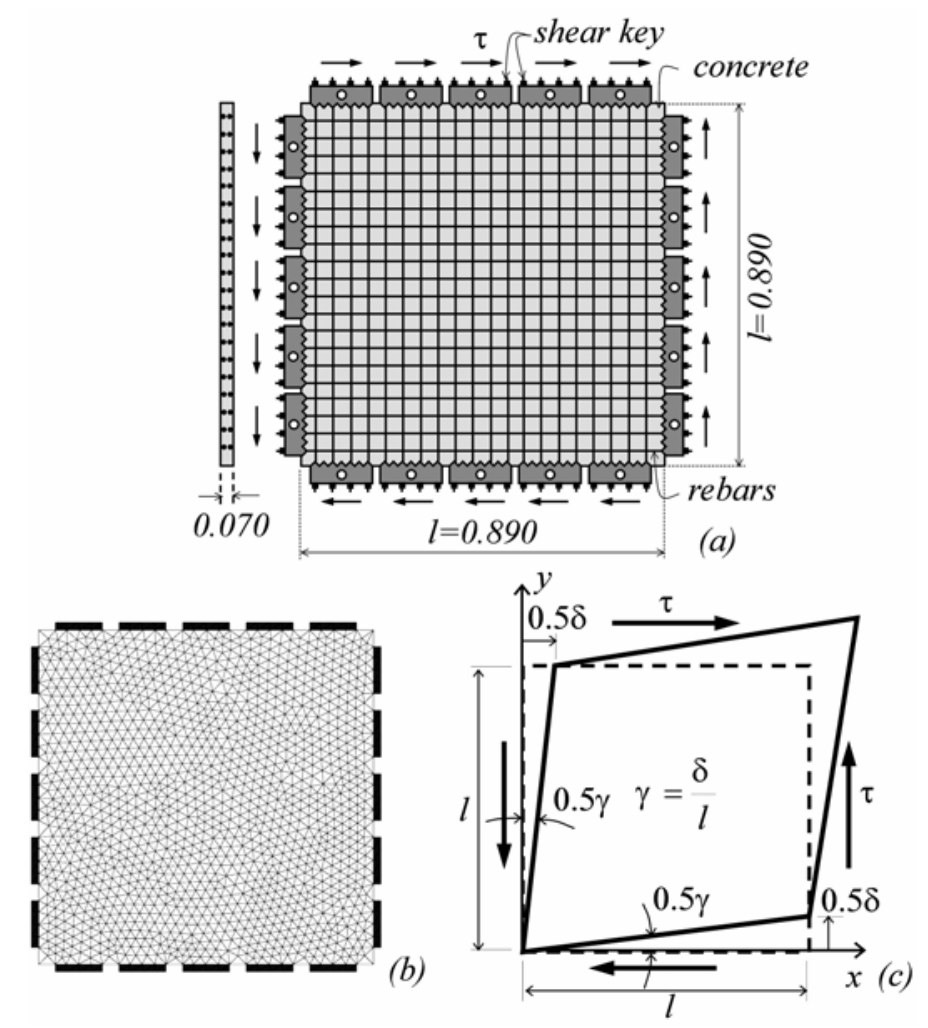

Figure 20. Reinforced concrete panel under shear: (a) geometrical description, (b) finite element mesh, (c) loading system for the numerical simulation.

The assumed material properties are the following: a) Concrete: Young's modulus $E^{m}=20 \mathrm{GPa}$, Poisson's ratio $v^{m}=0.2$, Fracture energy $G_{y}^{m}=100 . \mathrm{N} / \mathrm{m}$, tensile strength $\sigma_{u}^{m}=2 . \mathrm{MPa}$ and compression strength $\sigma_{u(c)}=20 . \mathrm{MPa}(n=10)$. b) Steel rebars: Young's modulus $E^{d}=200 \mathrm{GPa}$ and yield stress $\sigma_{y}^{d}=402 . \mathrm{MPa}$ (rebars are considered highly adherent so that bond slip effects at the interface are neglected). c) Dowel effect: elastic modulus $G^{f}=E^{d} / 2\left(1+v^{d}\right)=83.33 \mathrm{GPa}$, yield shear stress $\tau_{y}^{f}=\sigma_{y}^{d} / \sqrt{3}=263.3 \mathrm{MPa}$ and softening modulus $H^{f \tau}=0$.

Due to the very homogeneous distribution of the reinforcement, a uniform composite material model is assumed throughout the adopted finite element mesh shown in Figure 20b. The homogenous shear strain state, displayed in Figure 20c, has been applied in the numerical model. 


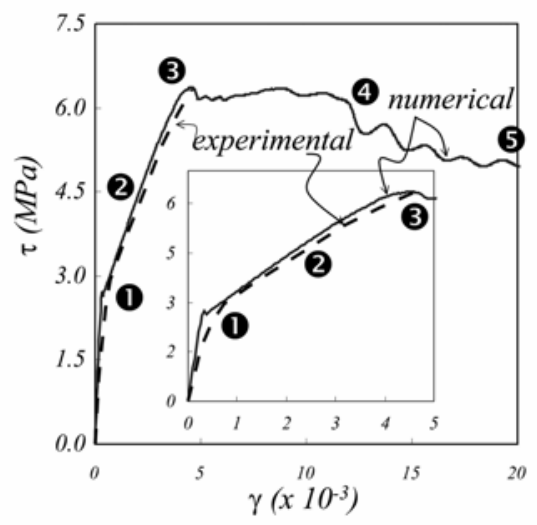

Figure 21. Reinforced concrete panel under pure shear stress. Structural shear stress vs. equivalent shear-strain curves.
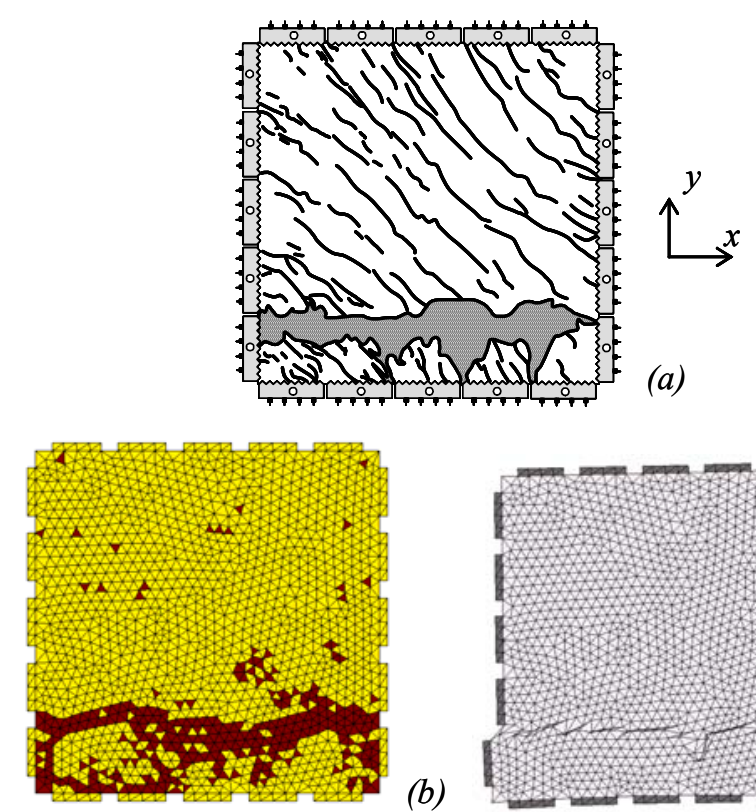

(b)

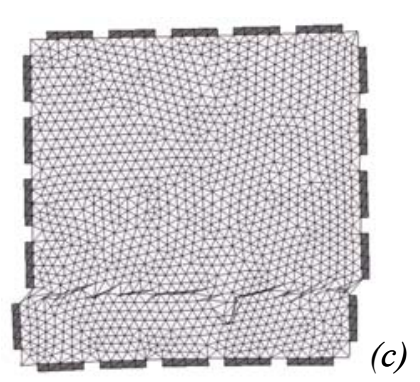

Figure 22. Reinforced concrete panel under pure shear. Results at the end of the analysis: (a) experimental crack patterns (Collins et al. [7]), (b) elements in inelastic loading (numerical active cracks) and (c) amplified deformed shape at the end of the simulation.

The numerical results are compared with the experimental ones in Figure 21. As it can be seen, numerical predictions agree very well with the experimental pre-critical branch of the structural behavior (points 1 to 3). Despite the inexistence of reported experimental results, the numerical simulation has been driven up to the very postcritical stages of the structural material failure (points 4 and 5).

Figure 22a displays the final crack pattern from the experimental test. At initial stages of the experiment, a distributed crack pattern, inclined 45 degrees with respect to the horizontal, was observed. These cracks, shown as solid lines in Figure 22a, correspond to the expected cracking in a concrete matrix subjected to a pure shear stress state, according to the maximum principal stresses failure criterion. In this early stage, no strength contribution from the rebars should be expected, since neither horizontal nor vertical stretching takes place. However, after the structural collapse, a very pronounced 
horizontal crack, displayed in grey in Figure 22a, was reported. This macro-crack formation, parallel to the reinforcement, was responsible for the sliding failure of the concrete panel.

As for the numerical simulation, the coalescence of a large number of inclined cracks, displayed by the active crack pattern (inelastic loading points) in Figure 22b, as well as the deformed (amplified) mesh in Figure 12c, demonstrate the good prediction of the reported collapse mechanism.

The numerical failure process is traced in Figure 23, where the displacement contours and the active crack patterns are displayed for different stages of the loading process marked in Figure 21. At the pre-critical stages 1 and 2, the distributed cracking pattern at 45 degrees translates into a complete spread of the active cracks over the panel, without concentration of the displacement contours. After stage 1, as the deformation increases, the dowel effect supplies additional strength to the composite material, giving rise to the hardening branch in Figure 21 (stages 2 and 3). Around stage 3, the yield shear stress in the rebars is attained and localization processes start, leading to horizontal fully developed cracks in stages 3, 4 and 5. These cracks correspond to the horizontal coalescence of some inclined distributed cracks, which remain in inelastic loading while those in their neighborhoods experience elastic unloading. As subsequent deformation develops (stages 4 and 5), some of those cracks get arrested and the final failure mechanism is characterized by the unique active macro crack shown in Figure 22.

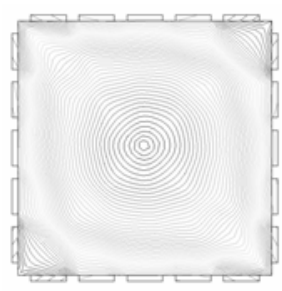

-

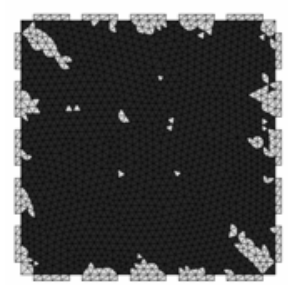

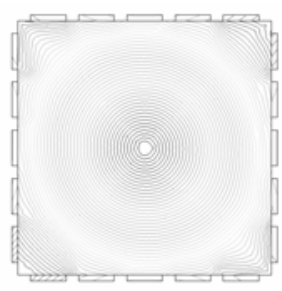

(2)

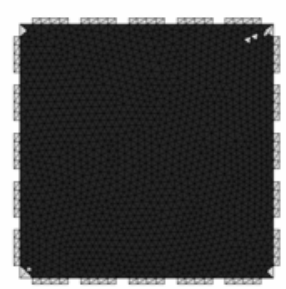

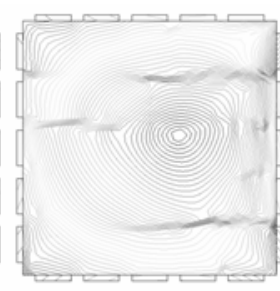

(B)

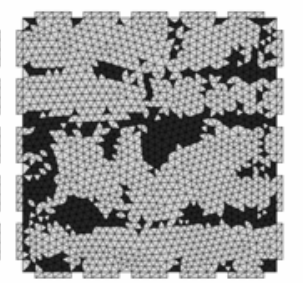

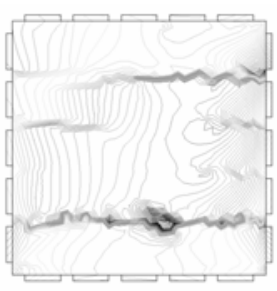

4

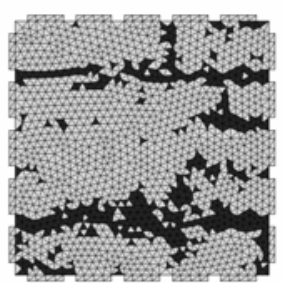

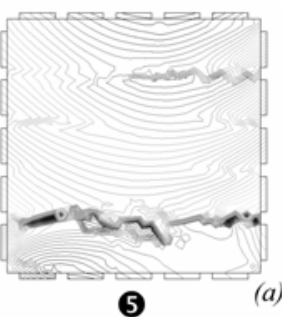

(5)

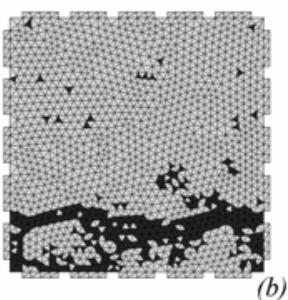

Figure 23. Reinforced concrete panel under pure shear stress: (a) Displacement contours for the different loading stages. (b) Active crack patterns (elements in inelastic loading are plotted in black).

\section{CONCLUDING REMARKS}

A new methodology to model reinforced concrete members has been proposed. It is based on the combination of the mechanical theory of mixtures and the framework of the CSDA using embedded crack finite elements.

The presented approach displays some advantages of using a continuum theory of material failure, in front of alternative discrete approaches based on traction separation laws. Actually, here the different individual phenomena, appearing in material failure of 
reinforced concrete, have been individually identified: concrete matrix failure, rebar mechanical failure, bond/slip effects and dowel action. Then, they have been separately modeled, on the basis of simple and familiar continuum dissipative models, and their relative contribution in the reinforced concrete has been accounted by using the classical mixture theory. The resulting continuum composite model can then be inserted in the CSDA framework, providing a material failure response for composite material accounting for all the aforementioned phenomena. Thus, there is no necessity of deriving a complex traction-separation law for such a complex material; it is implicitly and automatically fulfilled, in virtue of the projective properties of the CSDA, from the derived continuum composite model.

Therefore, the main attractive of the proposed approach is that, besides the individual mechanical behavior of the concrete and rebars, the most relevant interaction effects between those constituents are considered in the macroscopic representation of the composite provided by the mixtures theory. The dowel action and bond-slip effects can be introduced by means of simple one-dimensional elasto-plastic constitutive models, of easy characterization, applied to specific components of the macroscopic strain field. Then, the material failure, responsible for cracks formation and loss of strength in the composite material, can be treated using the CSDA in the same way than for a homogeneous material, but accounting for the participation of the constituents and their interactions.

The presented numerical simulations demonstrate the ability of the proposed methodology to predict the different stages of the mechanical behavior of tested reinforced concrete members, performed in distinct loading scenarios. The experimental curves of structural behavior, crack patterns and the collapse mechanisms can be reproduced with a remarkable accuracy.

The extension of the proposed methodology to three-dimensional problems, although not tackled here, seems to be quite straightforward.

\section{ACKNOWLEDGEMENTS}

Financial support from the Spanish Ministry of Science and Technology, trough grants BIA2005-09250-C03-03 and BIA2004-02080, is gratefully acknowledged. The fourth author acknowledges the sabbatical fellowship for foreign researchers (SAB2005-0060) from the Spanish Ministry of Science and Technology.

\section{APPENDIX}

\section{Damage model with distinct tensile and compressive strengths}

Consider the material model belonging to the family of damage models proposed by Simo and $\mathrm{Ju}[41]$, described by the following set of equations:

$$
\boldsymbol{\sigma}=\frac{q}{r} \bar{\sigma} ; \bar{\sigma}=\mathbf{C}: \varepsilon \quad \text { (constitutive relation) }
$$




$$
\begin{array}{ll}
f(\varepsilon, r)=\tau_{\varepsilon}-r \leq 0 ; & \text { (damage criterion) } \\
\tau_{\varepsilon}=\alpha \sqrt{\bar{\sigma}:(\mathbf{C})^{-1}: \bar{\sigma}}=\alpha \sqrt{\varepsilon: \mathbf{C}: \varepsilon} & \text { (softening law) } \\
\dot{q}=H \dot{r} & \text { (strain-like internal } \\
r(t)=\max _{s \in[0, t]}\left[r_{0}, \tau_{\varepsilon}(s)\right] ; r_{0}=\frac{\sigma_{u}}{\sqrt{E}} & \text { variable evolution) }
\end{array}
$$

where $\bar{\sigma}$ stands for the effective stress, $\mathbf{C}$ is the Hooke's elastic constitutive tensor, $q$ and $r$ are the stress-like and the strain-like internal variables, respectively, $H(<0)$ is the softening modulus, $\sigma_{u}$ is the tensile strength, $E$ is the Young's modulus and $\tau_{\varepsilon}$ is the norm of the strains in the metric defined by $\mathbf{C}$. As expressed by Equation (49), the strain-like internal variable, $r$, is the maximum historical value of the norm of the strains, $\tau_{\varepsilon}$, while the corresponding stress-like variable, $q$, is related to the strain-like one by means of the softening modulus, $H$, in Equation (48) (see references [22, 28] for additional information). Factor $\alpha$ is defined as:

$$
\alpha=\frac{\sum_{i=1}^{3}\left\langle\bar{\sigma}_{i}^{m}\right\rangle}{\sum_{i=1}^{3}\left|\bar{\sigma}_{i}^{m}\right|}\left(1-\frac{1}{n}\right)+\frac{1}{n}
$$

where $\bar{\sigma}_{i}$ is the $\mathrm{i}$-th principal effective stresses, $\langle\bullet\rangle$ is the Mac-Auley bracket and $n$ is the ratio between the compressive and tensile strengths.

\section{Constitutive tangent tensor}

The rate form of the constitutive relation (46) can be written as

$$
\dot{\boldsymbol{\sigma}}=\frac{q}{r} \mathbf{C}: \dot{\boldsymbol{\varepsilon}}+\frac{H r-q}{r^{2}} \dot{r} \overline{\boldsymbol{\sigma}}
$$

where the softening law of Equation (48) was used. In unloading cases, $\dot{r}=0$ and the constitutive relation reduces to

$$
\dot{\sigma}=\frac{q}{r} \mathbf{C}: \dot{\boldsymbol{\varepsilon}} \quad \text { (unloading case) }
$$

From Equations (47) and (49), in the loading case, the rate of the strain like variable is given by:

$$
\dot{r}=\dot{\tau}_{\varepsilon}=\left(\frac{r}{\alpha} \mathbf{C}:\left(\partial_{\overline{\boldsymbol{\sigma}}} \alpha\right)+\frac{\alpha^{2}}{r} \overline{\boldsymbol{\sigma}}\right): \dot{\boldsymbol{\varepsilon}}
$$


$\dot{\boldsymbol{\sigma}}=\left\{\frac{q}{r} \mathbf{C}-\frac{q-H r}{r^{3}}\left(\frac{r^{2}}{\alpha}\left(\overline{\boldsymbol{\sigma}} \otimes\left(\mathbf{C}: \partial_{\overline{\boldsymbol{\sigma}}} \alpha\right)\right)+\alpha^{2}(\overline{\boldsymbol{\sigma}} \otimes \overline{\boldsymbol{\sigma}})\right)\right\}: \dot{\boldsymbol{\varepsilon}}$ (loading case)

\section{Energy consumption analysis}

With the adopted regularized form of the CSDA, the energy consumption during the formation of the discontinuity is associated to the energy spent by the damage model in a localization band, $\Omega_{k}=k S \in \Omega$, of width $k \rightarrow 0$, with a median surface $S$, which undergoes inelastic strains in softening regime until reaching a complete material degradation (see .

The theorem of expended power states that, in the absence of kinetic energy (quasistatic case), the external power input in a solid, $P_{e x t}^{\Omega}$, equals the stress power, i.e.:

$$
P_{\text {ext }}^{\Omega}=\underbrace{\int_{\Omega} \sigma: \dot{\varepsilon} d \Omega}_{\text {stress power }}=\int_{\Omega} \frac{q}{r} \boldsymbol{\varepsilon}: \mathbf{C}: \dot{\varepsilon} d \Omega=\int_{\Omega} \frac{q}{r} \frac{\tau_{\varepsilon}}{\alpha^{2}} \dot{\tau}_{\varepsilon} d \Omega
$$

where the constitutive Equation (47) and the expression of $\tau_{\varepsilon}$ in equation (47) have been used.

That part of the external power internally spent in the formation of the discontinuity at the localization band $\Omega_{k}=k S \in \Omega$ is then:

$$
P_{\text {ext }}^{\Omega_{k}}=\int_{\Omega_{k}} \frac{q}{r} \frac{\tau_{\varepsilon}}{\alpha^{2}} \dot{\tau}_{\varepsilon} d \Omega_{k}=k \int_{S} \frac{q}{r} \frac{\tau_{\varepsilon}}{\alpha^{2}} \dot{\tau}_{\varepsilon} d \mathrm{~S}=k \int_{S} \frac{q}{\alpha^{2}} \frac{1}{H} \dot{q} d \mathrm{~S}
$$

where linear softening law and loading situation, in which $r=\tau_{\varepsilon}$, have been considered (see Equations (48) and (47)).

Therefore, the corresponding energy spent along any loading process leading the material from an unloaded initial state $\left(q=r_{0}\right.$ at $\left.t=0\right)$ to a complete degradation state $(q=0$ at $t=\infty)$ is given by:

$$
W^{S}=\int_{0}^{t_{\infty}} P_{e x t}^{\Omega_{k}} d t=\int_{0}^{t_{\infty}}\left[k \int_{S} \frac{q}{\alpha^{2}} \frac{1}{H} \dot{q} d \mathrm{~S}\right] d t=\int \underbrace{\left[k \int_{r_{0}}^{0} \frac{q}{\alpha^{2}} \frac{1}{H} d q\right]}_{S} d \mathrm{~S}=\int_{S}^{\left[\int_{G_{F}}^{0} \frac{q}{\left.r_{r_{0}} \frac{q}{\alpha^{2}} \frac{1}{\bar{H}} d q\right]}\right.} d S
$$

in which $\bar{H}=H / k$ is the so-called intrinsic softening modulus (see reference for more details).

The kernel of the last integral of Equation (57) is the energy spent per unit surface area in the formation of the crack, which is referred to as fracture energy, $G_{f}$, in the context of the non-linear fracture mechanics. 
If the intrinsic softening modulus, $\bar{H}$, is considered a material property independent of the loading process, the fracture energy for this damage model depends on the stress state, reflected by the factor $\alpha$.

In case of monotonic loading in uniaxial tension $\left(\bar{\sigma}_{1}>0, \bar{\sigma}_{2}=\bar{\sigma}_{3}=0, \alpha=1\right)$, the spent energy in Equation (57) corresponds to the mode I fracture energy:

$$
\begin{aligned}
& G_{f}^{I}=\frac{1}{\bar{H}} \int_{r_{0}}^{0} q d q=-\frac{r_{0}^{2}}{2 \bar{H}}=-\frac{\sigma_{u}^{2}}{2 E \bar{H}} \\
& \bar{H}=-\frac{\sigma_{u}^{2}}{2 E G_{f}^{I}}
\end{aligned}
$$

and the compressive failure energy spent in a monotonic loading in uniaxial compression $\left(\bar{\sigma}_{3}<0, \bar{\sigma}_{1}=\bar{\sigma}_{2}=0, \alpha=1 / n\right)$ can be written as:

$G^{c o m p}=n^{2} \frac{1}{\bar{H}} \int_{r_{0}}^{0} q d q=n^{2} G_{f}^{I}$

It is worth noting that the ratio $n$, for regular concrete, is about 10 , leading to a much more ductile behavior in compression than in tension, in accordance with experimental observations.

\section{REFERENCES}

[1] Arrea M.,Ingraffea A.R., Mixed-mode Crack Propagation in Mortar and Concrete, Dept. Struct. Eng. cornell Univ. New York 81-13, 1982.

[2] Bazant Z.P. Crack band theory for fracture of concrete. presented at Materiaux et Constructions, 1983.

[3] Belytschko T.,Moes N.,Usui S.,Parimi C. Arbitrary discontinuities in finite elements. International Journal for Numerical Methods in Engineering. 50, 9931013, 2001.

[4] Belletti B.,Cerioni R.,Iori I. Physical approach for reinforced-concrete (PARC) membrane elements. Journal of Structural Engineering. ASCE. 127, 1412-1426, 2001.

[5] Blanco S.,Huespe A.,Oliver J.,Pulido M.D.G. Strong discontinuity modeling of material failure in large concrete structures: recent computational developments and applications. presented at EURO-C 2006 Computational Modelling of Concrete Structures, Tyrol (Austria), 2006.

[6] Cervenka J., Mixed mode discrete crack propagation in concrete structures, vol. $\mathrm{Ph}$. D. Thesis. Boulder: University of Colorado, 1994.

[7] Collins M.,Vecchio F.,Mehlhorn G. An International competition to predict the response of reinforced concrete panels. Canadian Journal of Civil Engineering. 12, 624-644, 1985.

[8] Dvorkin E.N.,Cuitino A.M.,Gioia G. Finite elements with displacement embedded localization lines insensitive to mesh size and distortions.

Int.J.Num.Meth.Engng. 30, 541-564, 1990.

[9] Feenstra P.,de Borst R. Constitutive model for reinforced concrete. Journal of Engineering Mechanics - ASCE. 121, 587-595, 1995. 
[10] Hillerborg A. Numerical methods to simulate softening and fracture of concrete. in Fracture Mechanics of Concrete: Structural Application and Numerical Calculation, G. C. S. a. A. D. Tomaso, Ed., 1985, pp. 141-170.

[11] Jendele L.,Cervenka J. Finite element modelling of reinforcement with bond. Comp. Meth. Appl. Mech. Eng. 84, 1780-1791, 2006.

[12] Jirasek T.,Zimmermann T. Embedded crack model: I. basic formulation. International Journal for Numerical Methods in Engineering. 50, 1269-1290, 2001.

[13] Kollegger J.,Mehlhorn G. Material model for the analysis of reinforced concrete surface structures. Computational Mechanics. 6, 341-357, 1990.

[14] Leonhardt F. Reducing the shear reinforcement in reinforced concrete and slabs. Magazine of concrete research. 17, 187 - 198, 1965.

[15] Liao K.,Reifsnider L. A tensile strength model for unidirectional fiberreinforced brittle matriz composite. International Journal of Fracture. 106, 95 115, 2000.

[16] Lofti H.R.,Shing P.B. Embedded Representation of Fracture in Concrete with Mixed Finite-Elements. International Journal for Numerical Methods in Engineering. 38, 1307-1325, 1995.

[17] Manzoli O.L.,Shing P.B. A general technique to embed non-uniform discontinuities into standard solid finite elements. Computers \& Structures. 84, 742-757, 2006.

[18] Naaman A.,Namur G.,Alwan J.,Najm H. Fiber pullout and bond slip II. Experimental validation. Journal of Structural Engineering ASCE. 117, 27912800, 1991.

[19] Oliver J. A Consistent Characteristic Length for Smeared Cracking Models. International Journal for Numerical Methods in Engineering. 28, 461-474, 1989.

[20] Oliver J. Modelling strong discontinuities in solid mechanics via strain softening constitutive equations .1. Fundamentals. International Journal for Numerical Methods in Engineering. 39, 3575-3600, 1996.

[21] Oliver J. Modelling strong discontinuities in solid mechanics via strain softening constitutive equations .2. Numerical simulation. International Journal for Numerical Methods in Engineering. 39, 3601-3623, 1996.

[22] Oliver J. On the discrete constitutive models induced by strong discontinuity kinematics and continuum constitutive equa-tions. International Journal of Solid and Structures. 37, 7207-7229, 2000.

[23] Oliver J.,Cervera M.,Manzoli O. Strong discontinuities and continuum plasticity models: the strong discontinuity approach. International Journal of Plasticity. 15, 319-351, 1999.

[24] Oliver J.,Cervera M.,Oller S.,Lubliner J. Isotropic damage models and smeared crack analysis of concrete. presented at Proc. SCI-C Computer Aided Analysis and Design of Concrete Structures, 1990.

[25] Oliver J.,Huespe A.E. Continuum approach to material failure in strong discontinuity settings. Computer Methods in Applied Mechanics and Engineering. 193, 3195-3220, 2004.

[26] Oliver J.,Huespe A.E. Theoretical and computational issues in modelling material failure in strong discontinuity scenarios. Computer Methods in Applied Mechanics and Engineering. 193, 2987-3014, 2004. 
[27] Oliver J.,Huespe A.E.,Blanco S.,Linero D.L. Stability and robustness issues in numerical modeling of material failure in the strong discontinuity approach. Comput. Methods Appl. Mech. Engng. 195, 7093-7114, 2006.

[28] Oliver J.,Huespe A.E.,Pulido M.D.G.,Chaves E. From continuum mechanics to fracture mechanics: the strong discontinuity approach. Engineering Fracture Mechanics. 69, 113-136, 2002.

[29] Oliver J.,Huespe A.E.,Samaniego E. A study on finite elements for capturing strong discontinuities. International Journal for Numerical Methods in Engineering. 56, 2135-2161, 2003.

[30] Oliver J.,Huespe A.E.,Samaniego E.,Chaves E.W.V. On Strategies for Tracking Strong Discontinuities in Computational Failure Mechanics. presented at Fifth World Congress on Computational Mechanics (WCCM V), Vienna University of Technology, Austria, 2002.

[31] Oliver J.,Huespe A.E.,Samaniego E.,Chaves E.W.V. Continuum approach to the numerical simulation of material failure in concrete. International Journal for Numerical and Analytical Methods in Geomechanics. 28, 609-632, 2004.

[32] Ouyang C.,Shah P. Fracture energy approach for predicting cracking of reinforced concrete tensile members. ACI Structural Journal. 91, 69-78, 1994.

[33] Ouyang C.,Wollrab E.,Kulkarni S.,Shah P. Prediction of cracking response of reinforced concrete tensile members. Journal of Structural Engineering. ASCE. 123, 70 - 78, 1997.

[34] Pandolfi A.,Ortiz M. An efficient adaptive procedure for three-dimensional fragmentation Simulations. Engineering with Computers. 18, 148-159, 2002.

[35] Park R.,Paulay T. Reinfored Concrete Structures. Wiley ed, 1975.

[36] Pietruszczak S.,Winnicki A. Constitutive model for concrete with embedded sets of reinforcement. Journal of Engineering Mechanics - ASCE. 129, 725-738, 2003.

[37] Rots J.G.,Nauta P.,Kusters G.M.A.,Blaauwendraad J. Smeared crack approach and fracture localization in concrete. Heron. 30, 1-49, 1985.

[38] Ruiz G.,Elices M.,Planas J. Experimental study of fracture of lightly reinforced concrete beams. Materials and Structures. 31, 683-691, 1998.

[39] Simo J.,Oliver J.,Armero F. An analysis of strong discontinuities induced by strain-softening in rate-independent inelastic solids. Computational Mechanics. 12, 277-296, 1993.

[40] Simo J.C.,Hughes T.J.R. Computational Inelasticity: Springer, 1998.

[41] Simo J.C.,Ju J.W. Stress and strain based continuum damage models: I formulation. International Journal Solids and Structures. 15, 821-840, 1987.

[42] Tijssens M.G.A.,Sluys B.L.J.,van der Giessen E. Numerical simulation of quasibrittle fracture using damaging cohesive surfaces. European Journal of Mechanics a-Solids. 19, 761-779, 2000.

[43] Trusdell C.,Toupin R. The classical field theories. Handbuch der Physik III/I. Berlin: Springer Verlag, 1960.

[44] Wells G.N.,Sluys L.J. A new method for modelling cohesive cracks using finite elements. Int.J.Num.Meth.Engng. 50, 2667-2682, 2001.

[45] Xu X.P.,Needleman A. Numerical simulation of fast crack growth in brittle solids. J. Mech. Physics of Solids. 42, 1397-1434, 1994. 